\title{
HF radar observations of high-aspect angle backscatter from the E-region
}

\author{
S. E. Milan ${ }^{1}$, M. Lester ${ }^{1}$, T. K. Yeoman ${ }^{1}$, T. R. Robinson ${ }^{1}$, M. V. Uspensky ${ }^{2}$, and J.-P. Villain ${ }^{3}$ \\ ${ }^{1}$ Department of Physics and Astronomy, University of Leicester, Leicester LE1 7RH, UK \\ ${ }^{2}$ Finnish Meteorological Institute, Geophysical Research, FIN-00101, Helsinki, Finland \\ ${ }^{3}$ Centre National de la Recherche Scientifique, Orléans, F-45045, France
}

Received: 10 December 2002 - Revised: 21 July 2003 - Accepted: 29 July 2003 - Published: 19 March 2004

\begin{abstract}
We present evidence for the observation of highaspect angle HF radar backscatter from the auroral electrojets, and describe the spectral characteristics of these echoes. Such backscatter is observed at very near ranges where ionospheric refraction is not sufficient to bring the sounding radio waves to orthogonality with the magnetic field; the frequency dependence of this propagation effect is investigated with the Stereo upgrade of the CUTLASS Iceland radar. We term the occurrence of such echoes the "high-aspect angle irregularity region" or HAIR. It is suggested that backscatter is observed at aspect angles as high as $30^{\circ}$, with an aspect sensitivity as low as $1 \mathrm{~dB} \mathrm{deg}^{-1}$. These echoes are distinguished from normal electrojet backscatter by having low Doppler shifts with an azimuthal dependence that appears more consistent with the direction of the convection electric field $\boldsymbol{E}$ than with the expected electron $\boldsymbol{E} \times \boldsymbol{B}$ drift direction. This is discussed in terms of the linear theory dispersion relation for electrojet waves.
\end{abstract}

Key words. Ionosphere (ionospheric irregularities; plasma waves and instabilities; auroral ionosphere)

\section{Introduction}

Much work has been conducted over several decades in the study of coherent backscatter from plasma waves and irregularities in the auroral E-region, spanning radar observations in the HF, VHF, and UHF bands, that is scatter from irregularity scale sizes ranging from a few centimetres to $10 \mathrm{~s}$ of metres (see, for example, review papers of Haldoupis, 1989; Sahr and Fejer, 1996; Moorcroft, 2002). Most theoretical work suggests that irregularities should be highly aligned along the magnetic field direction, such that coherent backscatter can only be observed when radar signals propagate perpendicular to the magnetic field, known as the "orthogonality condition". However, there is a considerable

Correspondence to: S. E. Milan

(Steve.Milan@ion.le.ac.uk) body of evidence, especially in the UHF band, to suggest that irregularities exist which are inclined with respect to the magnetic field, that is scatter can be observed at angles away from orthogonality (Moorcroft, 1985, 1996; Schlegel and Moorcroft, 1989; Foster et al., 1992). The angle between the magnetic field normal and the direction of radar signal propagation is referred to as the "aspect angle" $\alpha$, and scatter significantly away from orthogonality (usually more than $1^{\circ}$ or $2^{\circ}$ ) is known as "high-aspect angle scatter".

At HF, and to a lesser degree VHF, refraction of the radar signals in the E-region allow for the orthogonality condition to be met routinely, and also makes it difficult to determine accurately the aspect angle if it is indeed non-zero. For these reasons, and from theoretical expectation, it is usually assumed that $\alpha=0^{\circ}$ where scatter is observed. Refraction of UHF radar signals is negligible and so the aspect angle of UHF backscatter can be calculated purely on the basis of the radar look-direction and magnetic field geometry. At these frequencies, backscatter is observed at aspect angles as high as $12^{\circ}$ or $13^{\circ}$ (Moorcroft, 1985, 1996), though backscatter power is found to fall off rapidly with increasing aspect angle. This "aspect sensitivity" is usually close to $\sim 10 \mathrm{~dB} \mathrm{deg}^{-1}$. UHF high-aspect angle echo spectra are found to be broad and range in Doppler shift from zero to in excess of the local ion-acoustic speed. The Doppler shift is thought to be approximately half of the line-of-sight component of the $\boldsymbol{E} \times \boldsymbol{B}$ drift (Schlegel and Moorcroft, 1989; Foster et al., 1992). Moorcroft (2002) has suggested that although a more expanded set of echo types have been attributed to high-aspect angle scatter at VHF, in fact most of these are probably a consequence of low-aspect angle scatter observed under conditions of high refraction in, for instance, intense sporadic E-layers. Neglecting such cases, true high-aspect angle VHF scatter has broadly the same characteristics as observed at UHF. So far, no concrete observations of high aspect angle scatter have been reported at HF.

In two recent papers (Milan and Lester, 1999, 2001) we identified five types of E-region echoes in HF radar backscatter (specifically from the Iceland East or CUTLASS 
Iceland radar of the SuperDARN (Greenwald et al., 1995) network), differentiated by their spectral characteristics such as Doppler shift and spectral width, together with flow angle behaviour. Populations (i) and (ii) appeared to have characteristics consistent with type I and II echoes observed at VHF (e.g. Haldoupis, 1989), though subsequent work has suggested that population (ii) has Doppler shifts significantly below expectations (Makarevitch et al., 2002; Milan et al., 2003a, b). Populations (iii) and (iv) have Doppler shifts above the ion-acoustic speed, and appear to have no direct analogue at VHF. The last echo population, population (v), we speculated, may arise due to scatter from irregularities which are not magnetic field-aligned. In the current paper we present evidence to support this hypothesis, and discuss it in terms of the propagation of HF radio waves in the ionosphere. As discussed by Moorcroft (2002), the unambiguous identification of backscatter from large aspect angles is difficult at VHF, let alone at HF, due to refraction. Key to understanding the propagation conditions responsible for the backscatter observed in our study is a multi-frequency experimental mode made possible by the new Stereo capability of the CUTLASS Iceland radar, first described by Milan et al. (2003a), and briefly re-introduced in Sect. 2. As described in Sect. 3.1, high-aspect angle backscatter is observed close to the radar where refraction is not sufficient to bring the radar signals orthogonal with the magnetic field; we term this the "high-aspect angle irregularity region" or HAIR. The propagation conditions that give rise to the HAIR echoes are discussed in Sect. 3.2. The Doppler shift characteristics of HAIR echoes, which are central to their identification, are found to differ considerably from the reported behaviour of high-aspect angle scatter at VHF and UHF, and are described and discussed in terms of the linear dispersion relation of electrojet plasma waves in Sect. 3.3. Finally, the spectral width and power characteristics of HAIR echoes are described in Sect. 3.4.

\section{The Stereo CUTLASS experiment}

The standard SuperDARN HF coherent scatter radar experimental arrangement and the "myopic" scan mode have been described in some detail before (Greenwald et al., 1995, and Milan and Lester, 2001, respectively), and only a brief summary will be given here. The radars are frequency-agile between 8 and $20 \mathrm{MHz}$, though for transmission licence considerations they are constrained to operate within a few allocated frequency bands within this range, each typically a few hundred $\mathrm{kHz}$ in width. The radars sound along 16 beam directions separated by $\sim 3.2^{\circ}$ of azimuth. In normal operation each beam is integrated for $7 \mathrm{~s}$, resulting in a scanning period of $2 \mathrm{~min}$, and is gated into 75 range cells of $45 \mathrm{~km}$ in length, with a range to the first cell of $180 \mathrm{~km}$. The radars transmit a 7-pulse scheme, which yields 17-lag autocorrelation functions, from which power, Doppler velocity, and spectral width can be determined (Villain et al., 1987; Hanuise et al., 1993). In addition, interferometric measurements made by a pair of antenna arrays allow the elevation angle $\Delta$ of the return echoes to be measured, which, in turn, gives a very approximate estimate of the altitude from which the echoes originate (Milan et al., 1997, 2001; Milan and Lester, 2001).

The Iceland East radar, one of the CUTLASS radars which form the eastern-most pair of the Northern Hemisphere SuperDARN chain, has been used extensively in the past for the study of E-region backscatter (Milan and Lester, 1998, 1999, 2001; Milan et al., 2000, 2001, 2003a, b). As part of these studies the "myopic" mode was developed in which the range separation is decreased to $15 \mathrm{~km}$, to give high spatial resolution observations of the near-range field-of-view, that portion of the field-of-view in which E-region echoes are routinely detected. Usually the myopic mode has been run with a radar operating frequency of $10 \mathrm{MHz}$, and a beam dwell period of $3 \mathrm{~s}$. The Iceland East radar, located at Thykkvibær, Iceland $\left(63.77^{\circ} \mathrm{N}, 339.46^{\circ} \mathrm{E}\right)$, with a boresite azimuth of $30^{\circ}$ east of north, is again employed in the present study.

Recently, the versatility of the CUTLASS radars has been enhanced by the "Stereo" upgrade, which effectively turns each radar into two. In normal SuperDARN operation only half of the available transmitter duty-cycle $(\sim 7 \%)$ is used to generate the radar pulse sequence. Stereo exploits the remaining unused duty-cycle by interleaving two pulse sequences at different radar frequencies. Then, by employing the original, plus an additional receiver system, it is possible to sound simultaneously on two radar "channels", known as A and B. The beams of the two channels can be steered independently, and in principle, the range separation or range to the first cell of each channel can also differ. Routine operation of the Stereo capability of CUTLASS commenced in January 2002, though test runs were conducted in the latter half of 2001. In December 2001 the myopic mode exploited Stereo to make multi-frequency observations of the E-region, and it is the Stereo myopic run on the night of the 14-15 December, 18:00-06:00 UT, that is the focus of the present study. Channel A operated in myopic mode as normal, at a radar frequency of $10 \mathrm{MHz}$, completing a scan of the field-ofview every $63 \mathrm{~s}$. Channel B sounded each channel A beam simultaneously, though at a different frequency; each scan of channel B was conducted at one of 5 frequencies, in order $8,12,14,16$, and $18 \mathrm{MHz}$, with a complete cycle being completed every $315 \mathrm{~s}$.

In addition to the Iceland East radar we also employ the Iceland West radar located at Stokkseyri, just a few km to the west of Thykkvibær. This radar has a boresite azimuth of $59^{\circ}$ west of north, thus providing a large range of azimuthal lookdirections when taken together with the Iceland East radar. Both radars were occasionally operated together in myopic mode in 1999 and 2000, and observations from some of these runs will be used to study the azimuthal behaviour of the Eregion backscatter. Hereupon, we will refer to the Iceland East and West radars as IE and IW, respectively. 


\section{Observations and discussion}

\subsection{Multi-frequency observations of HAIR echoes}

Milan and Lester $(1999,2001)$ presented evidence that five populations of E-region HF echoes exist, using Doppler shift, spectral width and return power characteristics. In general, the Doppler shift imposed on echoes is depressed below the line-of-sight component of the electron drift, though in the main the sign of the Doppler shift (towards or away from the radar) is consistent with the direction expected for zonal electron drift in the eastward or westward electrojets (Milan et al., 2003a), i.e. for the eastward-pointing IE radar most Doppler shifts are positive (towards the radar) and negative (away from the radar) in the eastward and westward electrojets, respectively. (IE typically observes eastward and westward electrojet backscatter between magnetic local times of 18:00 to 00:00 MLT and 00:00 to 06:00 MLT, respectively; $\mathrm{MLT} \approx \mathrm{UT}$ for the near-range IE field-of-view.) However, one of the five characteristic populations of echoes observed is identified primarily as having Doppler shifts which contravene this rule. Figure 1 shows examples of radar scans containing such echoes, one case from the eastward electrojet ( 20:05 UT, 14 December 2001) and the other from the westward electrojet ( 02:45 UT, 15 December 2001). Five consecutive scans are shown in each example, with simultaneous observations made on the two Stereo channels for each scan. The top row of each pair of panels indicates channel A observations made at $10 \mathrm{MHz}$ in each scan, the bottom panels showing channel B observations made at 8, 12, 14,16 , and $18 \mathrm{MHz}$ in turn. Elevation angle measurements from the radar interferometer show that in both examples the main body of backscatter at the nearer ranges (generally gates 45 and below, slant ranges less than $850 \mathrm{~km}$ ) originates from the E-region; at further ranges the backscatter comprises F-region echoes. The E-region backscatter is curved in form, a consequence of sounding an L-shell-aligned scattering region with a radar that sweeps in azimuth from slightly east of north (beam 0 , magnetic azimuth $23^{\circ}$ ) to slightly north of east (beam 15 , magnetic azimuth $73^{\circ}$ ), as shown in Figs. 5b and c. Within the backscatter region, L-shellaligned structure in the Doppler shift can be discerned, especially in the eastward electrojet; similar features are also present in backscatter power and spectral width. Also, as mentioned above, most echoes in the eastward electrojet have positive Doppler shifts, with negative Doppler shift echoes predominating in the westward electrojet. Of most interest to this study are the regions of echoes at the very nearest ranges, in general closer than range gate 15 (slant range $400 \mathrm{~km}$ ), in which low but oppositely-directed Doppler shifts are observed. These echoes are population (v) identified by Milan and Lester (2001). In Sect. 3.2 we will argue that these echoes scatter from non-field-aligned irregularities, or in other words, irregularities with high aspect angles. As a consequence, we will refer to such backscatter as the "highaspect angle irregularity region", or HAIR.
The most striking feature of these echoes is that although the backscatter region as a whole is L-shell aligned, the boundary between normal and HAIR echoes occurs at uniform range, irrespective of look-direction (radar beam). In channel $\mathrm{A}$, at $10 \mathrm{MHz}$, this boundary occurs near range gate 13 (slant range $375 \mathrm{~km}$ ) in the first example, and range gate 10 (slant range $330 \mathrm{~km}$ ) in the second example. In channel $\mathrm{B}$, where frequency changes from scan to scan, the range at which this boundary is observed increases with increasing frequency. The uniform range with look-direction and the increase of range with frequency suggest that the observation of this echo population is controlled by the propagation of the radar signal, that is its level of refraction in the E-region ionosphere. In other words, the boundary between normal and HAIR echoes appears to be a consequence of a change in scattering geometry, and does not represent a geophysical boundary between different irregularity types.

Figure 2 shows the Doppler shift, SNR or backscatter power, spectral width, and elevation angle of echoes for all beams as a function of range gate in channel $\mathrm{A}(10 \mathrm{MHz})$ for the intervals (a) 20:00-20:30 UT, 14 December, and (b) 02:40-03:10 UT, 15 December, half-hour periods during which the HAIR features persist. Superimposed in red are median values in each range gate, with horizontal bars showing the upper and lower quartile values. At further ranges Doppler shifts are positive (Fig. 2a) and negative (Fig. 2b) as expected for scatter in the eastward and westward electrojets. At these ranges echoes fall into two main populations, those with higher $\left(\left|v_{\text {los }}\right|>300 \mathrm{~m} \mathrm{~s}^{-1}\right)$ and lower $\left(\left|v_{\text {los }}\right|<300 \mathrm{~m} \mathrm{~s}^{-1}\right)$ Doppler shifts. This mixture of high and low velocity echoes was identified as characteristic of E-region backscatter by Villain et al. (1987, 1990), Makarevitch et al. (2002), and Milan et al. (2003a, b). Not only can echoes belong to one or the other population, but individual echoes can contain two spectral components, one with high and the other with low Doppler shift. For this reason Milan et al. (2003a) termed these "two peak E-region echoes", or TWOPEEs. The spectral forms of such echoes will be examined in more detail in Sect. 3.4. Whether the median Doppler shift falls in the high Doppler shift population (Fig. 2a) or low Doppler shift population (Fig. 2b) depends on the relative occurrence of the echo types. Irrespective of which echo type dominates at further ranges, in both Figs. $2 a$ and $b$ there is a clear transition between reverse- to normal-sense Doppler shifts near range gates 13 and 10 (slant ranges 375 and $330 \mathrm{~km}$ ), respectively, though with a slight overlap of one or two range gates between the two populations. We call the range at which this Doppler shift reversal occurs $d_{v e l}$.

In the very nearest range gates (two or three), very few echoes are observed. Here, however, Doppler shifts return to the expected sense, though with very low magnitudes, and spectral widths become very low (see esp. example b). We identify these echoes, which appear as the "grainy near-range echoes" described by Hall et al. (1997), as meteor scatter from D-region altitudes (see below), the Doppler shifts giving the line-of-sight component of the neutral wind speed. 
a Eastward electrojet, 14 December 2001
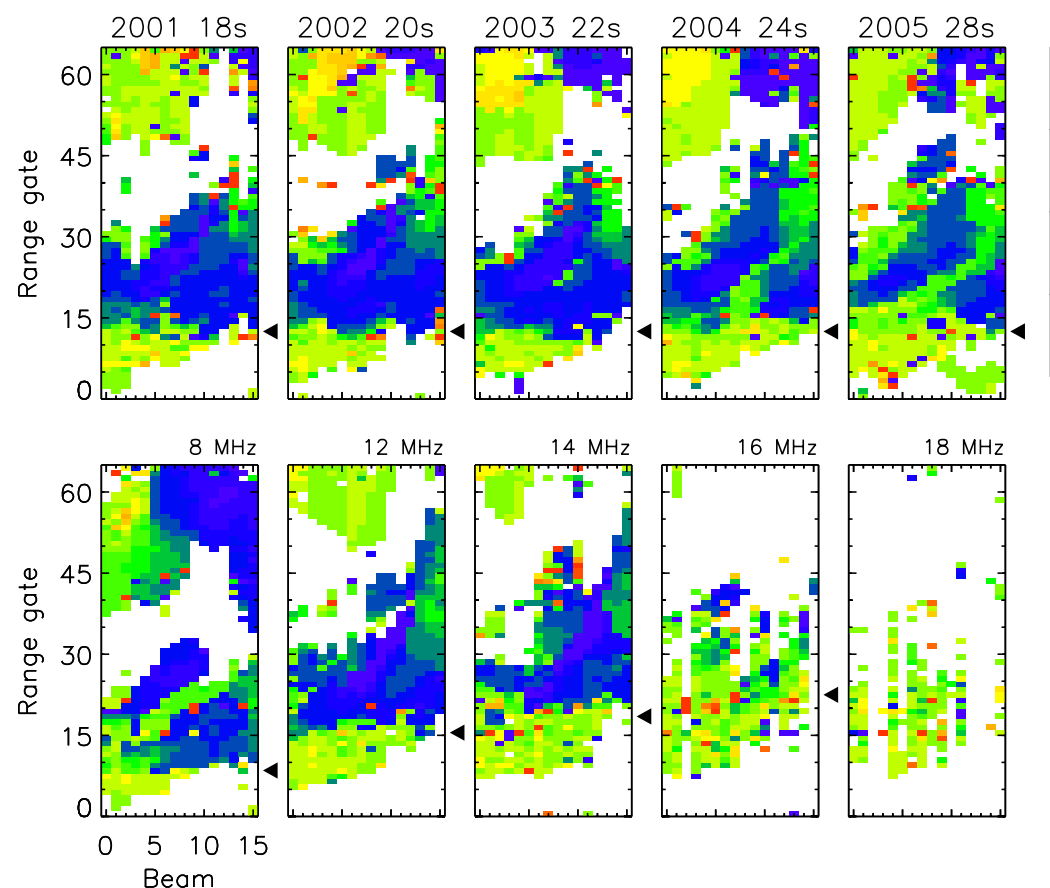

\section{b Westward electrojet, 15 December 2001}
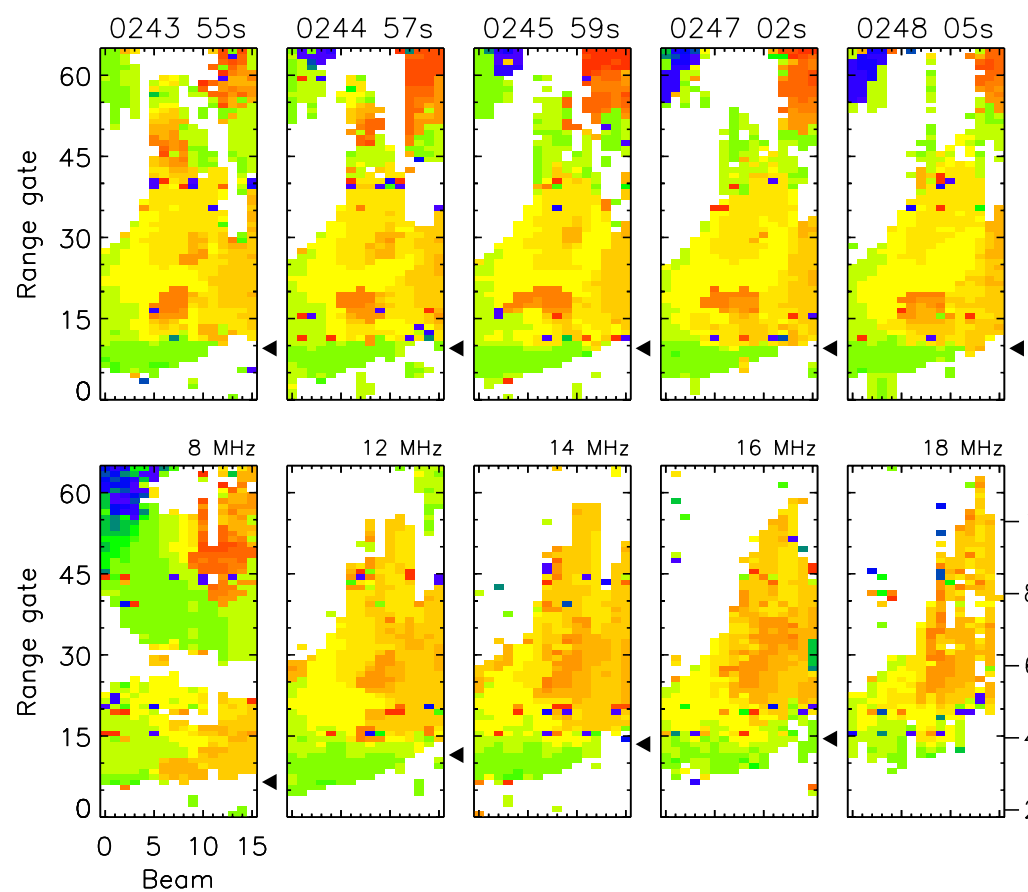

Fig. 1. Examples of the occurrence of HAIR echoes in HF radar electrojet backscatter. Two groups of panels show radar scans in the eastwards electrojet (20:01-20:06 UT, 14 December 2001) and westwards electrojet (02:4402:49 UT, 15 December 2001). In each case upper panels present soundings at $10 \mathrm{MHz}$, whereas lower panels show simultaneous soundings at $8,12,14,16$, and $18 \mathrm{MHz}$.
Turning to the backscatter power variation, there is a characteristic increase in SNR with range to some maximum, beyond which SNR falls again. In both cases the power maximum occurs several range gates beyond the velocity transition. Backscatter power measurements are often difficult to interpret as they depend not only on backscatter cross section, but also on vertical antenna gain, focusing and defo- cusing of the radar beam in the ionosphere, and attenuation in the D-region. However, for geometrical reasons power is usually expected to decrease with increasing slant range, $d$ (e.g. Hunsucker, 1991). Thus, the near-range increase in power is contrary to expectations, and we identify the range of the SNR maximum as the limit of the HAIR region, $d_{\text {HAIR }}$. In Sect. 3.4 we will discuss this variation in power in terms 

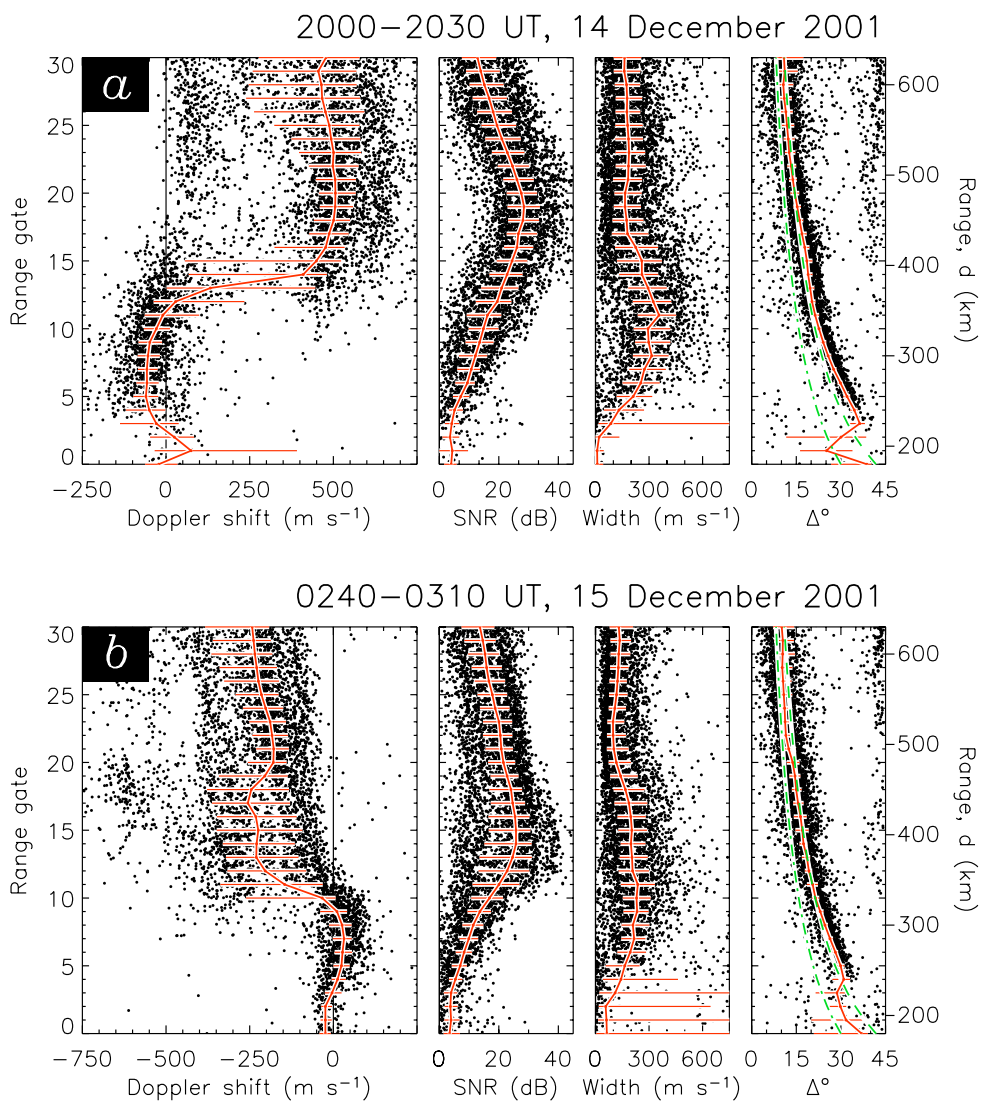

Fig. 2. Echo characteristics as a function of range in the eastwards (20:00-20:30 UT) and westwards (02:40-03:10 UT) electrojets at $10 \mathrm{MHz}$; the red line and bars indicate the median and upper and lower quartiles. Left-hand panels present Doppler shift; the region of HAIR echoes at near ranges is clearly discernable. Towards the right panels show backscatter power, spectral width, and elevation angle, $\Delta$. Dashed and dot-dashed green curves indicate $\Delta$ predictions for virtual heights of 120 and $90 \mathrm{~km}$, respectively.

of a range variation in the aspect angle of the irregularities from which the radar scatters.

Throughout the range of observations shown, the elevation angles follow closely the predictions of $\Delta$ for a scattering layer with a "virtual height" (see Sect. 3.2) near $120 \mathrm{~km}$ (dashed green curves), except in the nearest two or three range gates. Here the elevation angles are consistent with a lower altitude (dot-dashed green curves indicate the expectation for $90 \mathrm{~km}$ ), as expected for meteor scatter.

Figure 3 shows median curves of Doppler shift (red) and SNR (blue) determined for all the frequency bands sounded between 8 and $16 \mathrm{MHz}$, upper and lower panels for the eastward and westward electrojet examples, respectively. As suggested by Fig. 1 echoes can be somewhat sparse in the $18 \mathrm{MHz}$ frequency band, and as a consequence, we concentrate on the five lower bands -8 to $16 \mathrm{MHz}$ - in the rest of this paper. In each band, the red and blue triangles indicate the ranges of the Doppler shift transition, $d_{v e l}$, and the power maximum, $d_{\text {HAIR }}$, respectively, where these can be determined. In both electrojets $d_{v e l}$ and $d_{\text {HAIR }}$ increase significantly with increasing frequency. This is also demonstrated in Fig. 4 , in which $d_{v e l}$ and $d_{\text {HAIR }}$ (red and blue curves, respectively) are shown as a function of frequency, the dashed and full curves determined for the eastward and westward electrojet examples. Error bars of \pm 1 range gate are shown to indicate the overlap between normal and reverse echoes near $d_{v e l}$, and the slight uncertainty in determining $d_{\text {HAIR }}$. In addition to the increase in $d_{v e l}$ and $d_{\text {HAIR }}$ with frequency, we find in these examples a very close relationship between the two, such that $d_{\mathrm{HAIR}} \approx 1.25 d_{\text {vel }}$. We now investigate the frequency dependence of $d_{\text {HAIR }}$ and discuss the implications in terms of propagation considerations.

\subsection{Propagation considerations and an explanation of HAIR echoes}

For coherent radars to sense backscatter from field-aligned density perturbations or irregularities the transmitted radio wave must propagate orthogonal to the magnetic field at the point of scatter. This geometry can only be achieved within a relatively limited field-of-view by a VHF radar, as its transmissions - at frequencies usually in excess of $50 \mathrm{MHz}-\mathrm{ex}$ perience little refraction in the lower ionosphere. In contrast, an HF radar relies on the ionosphere to refract the transmitted radio waves $(\sim 10 \mathrm{MHz})$ to orthogonality with the magnetic field, so that it can sense backscatter from a much larger viewing area. The level of refraction is dependent on the electron density within the ionosphere, specifically in the Eregion for backscatter from the electrojets. The undisturbed nighttime E-region electron density is typically of the order of $5 \times 10^{9} \mathrm{~m}^{-3}$ (Davies, 1990). In terms of plasma frequency, where plasma frequency is related to electron density by

$f_{p}^{2}=\frac{N_{e} e^{2}}{4 \pi^{2} \varepsilon_{0} m_{e}}$, 

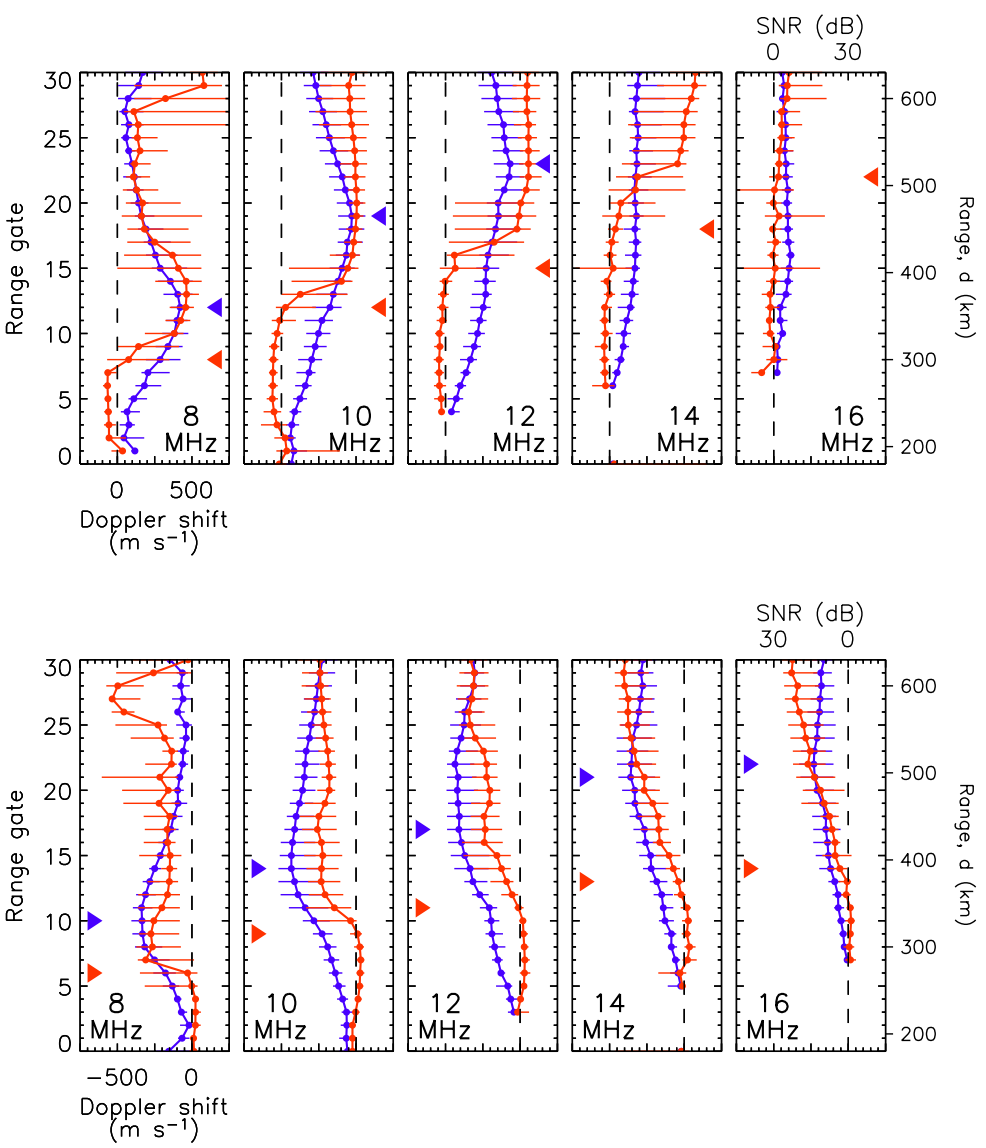

Fig. 3. Similar to Fig. 2, though showing the variation of Doppler shift (red) and SNR (blue) characteristics with increasing frequency, $8,10,12,14$, and $16 \mathrm{MHz}$. Red and blue triangles indicate $d_{v e l}$ and $d_{\text {HAIR }}$, respectively. where the symbols have their usual meanings, this is equivalent to $f_{p} \approx 0.6 \mathrm{MHz}$. For radar soundings at frequencies near $10 \mathrm{MHz}$ this gives rise to negligible refraction, and consequently, no backscatter from field-aligned irregularities can be observed. Moreover, in these undisturbed regions of the ionosphere few irregularities are found, so no backscatter should be expected.

In the auroral zones, however, particle precipitation leads to an enhancement of the E-region electron density, typically to values of 1 or $2 \times 10^{11} \mathrm{~m}^{-3}$, that is plasma frequencies of 3 to $4 \mathrm{MHz}$ (e.g. Whalen, 1983). These enhanced electron densities result in elevated Hall and Pedersen conductivities which allow large currents - the electrojets - to flow. In addition, these regions abound in the ionospheric irregularities from which radar signals scatter, and the electron density is sufficiently high to provide the refraction necessary for backscatter to be observed. All HF radar echoes that are observed are usually interpreted as scatter from irregularities that are very close to field-aligned (aspect angles $\alpha \leq 1^{\circ}$ ). However, from an understanding of HF propagation we show that the HAIR population described above appears inconsistent with this interpretation.

At this point we recap briefly the major features of HF propagation in the ionosphere; a much fuller description of HF propagation can be found in Davies (1990). Figure 5a shows a schematic of four rays (wave vector $\boldsymbol{k}$ ) launched at a range of elevation angles $\Delta$ from the radar. These rays are labeled $\mathrm{A}$ to $\mathrm{D}$ in order of increasing $\Delta$; in the following discussion we focus on ray $\mathrm{B}$. In our analysis we make two main assumptions: that the curvature of the Earth can be neglected, approximately valid for the short ranges under consideration, and that the Earth's magnetic field points vertically downwards at these polar latitudes. Two horizontal dashed lines mark the altitudes of the bottom and the peak of electron density in the E-region. Above the peak the electron density is expected to fall again until the bottomside of the F-region is encountered. We also indicate that the elevated electron density of the E-region is limited in spatial extent, and tends to a maximum near the centre of the electrojet, falling at higher and lower latitudes (Whalen, 1983).

The radar ray propagates in a straight line until it reaches the E-region (assuming negligible D-region refraction), with an angle of incidence $\zeta$. Within the E-region the increasing electron density causes the ray-path to be refracted. If the peak electron density or critical plasma frequency of the E-region is sufficient that the ray is refracted to the horizontal, then the ray is "reflected" back towards the ground. Because the Earth's magnetic field is assumed to be nearly vertical, the point at which the ray is perpendicular to the magnetic field (that is, the point at which backscatter from field-aligned irregularities can be observed, double arrow) necessarily occurs near the point of reflection. 


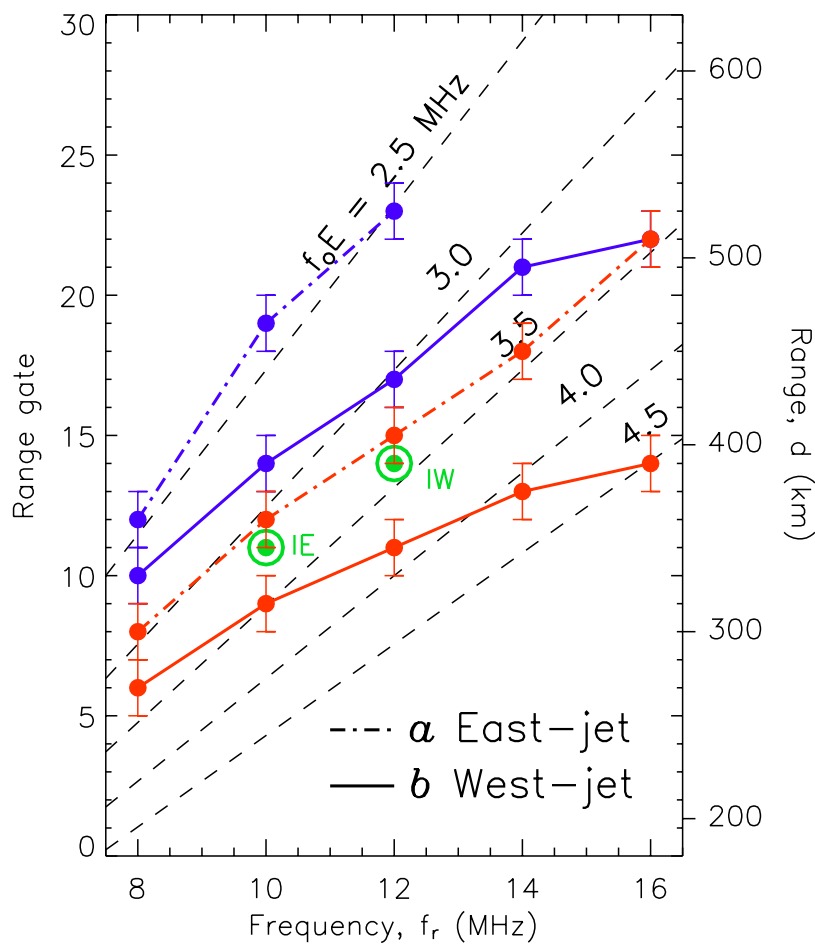

Fig. 4. The variation of $d_{v e l}$ (red) and $d_{\text {HAIR }}$ (blue) with frequency band for the eastwards (20:00-20:30 UT) and westwards (02:40 03:10 UT) electrojets (dot-dashed and solid curves, respectively). Superimposed are predications of $d_{\min }$ for E-region critical frequencies of $f o \mathrm{E}=2.5,3.0,3.5,4.0$, and $4.5 \mathrm{MHz}$. Also indicated by green dots are $d_{\text {HAIR }}$ versus $f_{r}$ for the IW and IE observations presented in Figs. 10 and 11.

For simplicity the true ray path can be represented by the "virtual path" - the triangular dotted line - which assumes straight-line propagation and plane reflection. This path has a "virtual height", $h^{\prime}$, which is greater than the true reflection height. When estimating altitude from interferometer measurements of $\Delta$ we implicitly mean virtual, not true, height as we must assume straight line propagation, having no accurate knowledge of the level of refraction occurring in the ionosphere. However, if the E-region is assumed to be thin, then this discrepancy will not be too considerable; we have used a virtual height of $120 \mathrm{~km}$ as opposed to a true height of 100 to $110 \mathrm{~km}$ in our $\Delta$ predictions in Fig. 2. The length of the side of the virtual path, $d$, is equivalent to the timeof-flight of the ray to the true reflection point multiplied by the speed of light ("Breit and Tuve's theorem", see Davies, 1990). This is so because, although the true path is shorter than the virtual path, the ray is slowed within the ionosphere. If backscatter is observed from near the point of reflection, then the slant range calculated from the time-of-flight of the echoes will be $d$. Backscatter can also be observed from the ground incidence of the reflected ray; this should be observed at a range of $2 d$. Note that the same incoming elevation angle $\Delta$ would be measured by the radar for both the ionospheric and ground backscatter generated by this ray.
A vertically-propagating ray reflects at the altitude at which the radar frequency, $f_{r}$, matches the plasma frequency, $f_{p}$; if the radar frequency exceeds the peak E-region plasma frequency - known as the E-region critical frequency or $f o \mathrm{E}$ ("o" for ordinary-mode) - then the ray is not reflected, is said to penetrate the E-region, and continues upwards into the F-region. For a ray propagating obliquely a similar situation holds, except that reflection occurs at a plasma frequency such that

$f_{r}=f_{p} \sec \zeta$

known as the "secant law" (see Davies, 1990), a consequence of Snell's Law. In other words, the more oblique a ray, the greater the radar frequency that a given E-region will reflect. The corollary of this is that at a given radar frequency, there is a minimum value of $\zeta$ (and a maximum value of $\Delta$ ) that will lead to reflection; rays less oblique than this will penetrate the E-region (e.g. rays $\mathrm{C}$ and D shown in Fig. 5a). The limiting $\zeta_{\min }$ (and $\Delta_{\max }$ ) occurs at the point that the apex of the true path coincides with the altitude of the peak of the Eregion, as is the situation for ray B. At $\Delta_{\max }$ the slant range to the point of orthogonality is a minimum, which we call $d_{\min }$. For such a ray, the ground range from the radar to the point of ground incidence is also a minimum, and is known in HF parlance as the "skip distance". The slant range to the ground scatter is also a minimum, which we call $d_{\text {skip }}$, and is equal to $2 d_{\min }$.

We have also indicated the paths of two rays which penetrate the E-region: they are refracted within the enhanced electron density, but not sufficiently to cause reflection. In this case, the rays are not brought to the horizontal, and as a consequence, do not reach orthogonality with the magnetic field. In what follows we hypothesize that normal echoes are observed where orthogonality with the field can be achieved (rays A and B), whereas at closer ranges orthogonality is not reached and HAIR echoes are detected (rays C and D). If this is the case, then we expect our observed transition range between normal and HAIR echoes, $d_{\text {HAIR }}$, to correspond to the minimum range at which orthogonality can theoretically be achieved, $d_{\min }$. For a roughly L-shell-aligned electrojet region (Fig. 5b) the location of E-region echoes should progress from nearer to further ranges with increasing azimuth from north (Fig. 5c), with HAIR echoes appearing in beams where scatter is observed at ranges closer than $d_{\text {min }}$.

In our idealized flat-Earth picture, the relationships between the key parameters are simple. The virtual height, radar range, and angles of elevation and incidence are related by

$h^{\prime}=d \sin \Delta=d \cos \zeta$

as $\zeta=90^{\circ}-\Delta$. We can use Eq. (3) to calculate the expected elevation angle $\Delta$ as a function of range $d$ for a given virtual height $h^{\prime}$, as in Fig. 2. From Eqs. (2) and (3),

$f_{p}=f_{r} \frac{h^{\prime}}{d}$, 

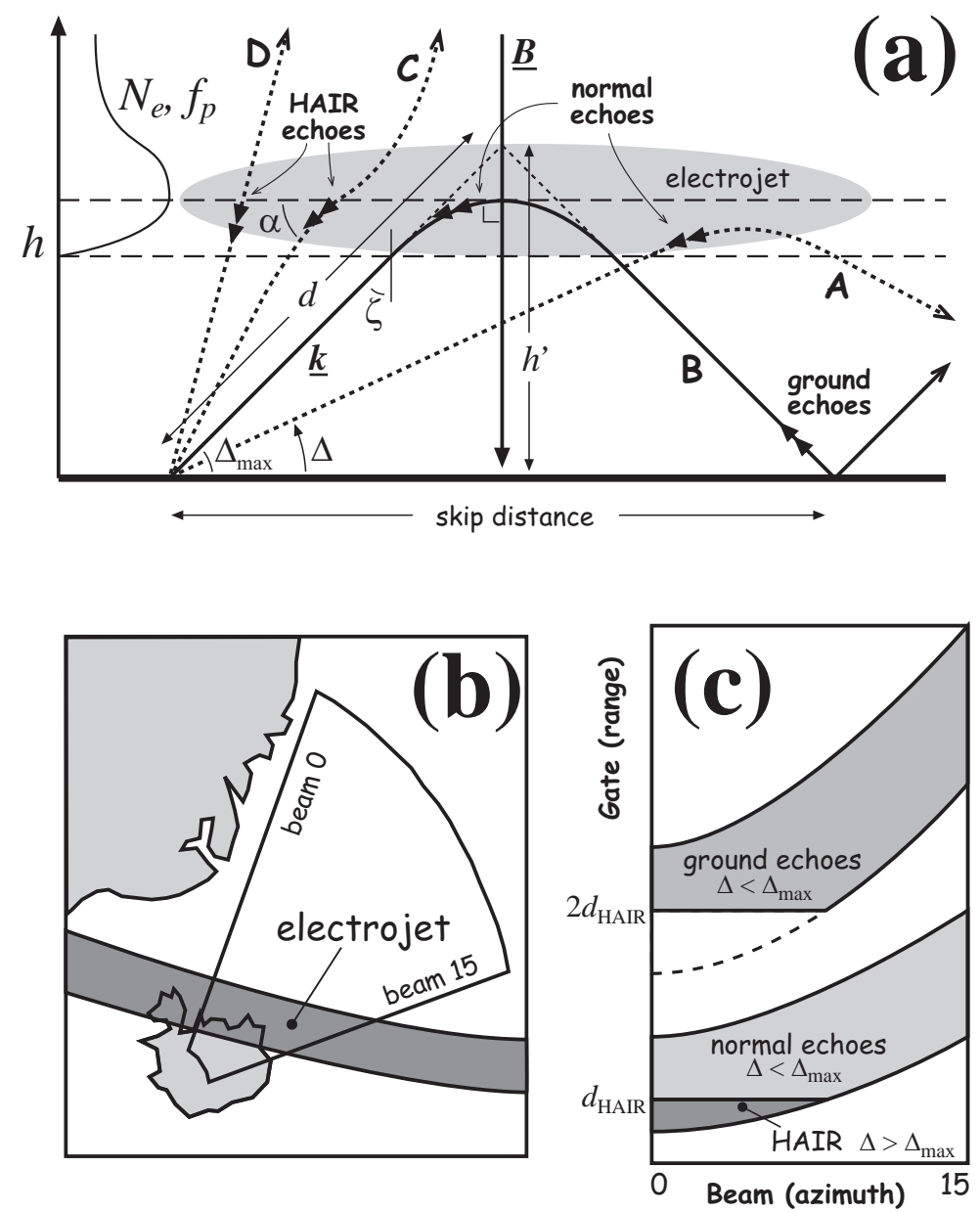

Fig. 5. (a) A schematic figure showing the expected propagation geometry of HF radio waves in the electrojet region (see text for details). Double arrows show the locations from which backscatter might be seen, whether normal E-region echoes, ground echoes, or HAIR echoes. (b) A typical orientation of the electrojet relative to the IE radar, and (c) the resulting pattern of HAIR, normal, and ground echoes within the field-of-view. which gives the plasma frequency at which the radar signal is reflected as a function of range, $d$. If this required $f_{p}$ exceeds $f o \mathrm{E}$, then the ray is not reflected. Hence, knowing the nearest range at which a ray reaches orthogonality with the magnetic field for a given radar frequency, $d_{\min }\left(f_{r}\right)$, allows us to estimate the critical frequency of the E-region (assuming spatial uniformity), from Eq. (4):

$f o \mathrm{E}=f_{r} \frac{h^{\prime}}{d_{\min }\left(f_{r}\right)}$,

or, equally,

$d_{\min }\left(f_{r}\right)=h^{\prime} \frac{f_{r}}{f o \mathrm{E}}$.

As $f o \mathrm{E}$ is constant for a given E-region and $h^{\prime}$ should not vary too much as a function of frequency for a thin E-region, this allows us to determine the frequency-dependence of the minimum range $d_{\min }$ from which normal backscatter should be observed. Dashed black curves showing this relationship are superimposed on Fig. 4 for different values of $f o \mathrm{E}$. We expect that our empirical (blue) curves of $d_{\text {HAIR }}$ should roughly follow the model curve of $d_{\min }$ appropriate for the critical frequency of the E-region in either case. Although we have no knowledge of $f o \mathrm{E}$ over Iceland at the times of the observations, we find that the frequency variation of $d_{\text {HAIR }}$ is roughly consistent with values of $f o \mathrm{E}$ near 2.5 and $3 \mathrm{MHz}$ in the eastward and westward electrojets examples, respectively, with both values typical of the nightside auroral zone E-region (Whalen, 1983).

To summarize, the variation of $d_{\text {HAIR }}$ appears to suggest that normal echoes are observed where orthogonality between the radar rays and the magnetic field can be achieved; the HAIR echoes observed at nearer ranges appear to originate from a similar altitude as the normal echoes (see $\Delta$ variation in Fig. 2), but from regions where orthogonality cannot be achieved. In other words, HAIR echoes appear to arise as a consequence of high-aspect angle scatter. In what follows we use the terms $d_{\min }$ and $d_{\text {HAIR }}$ somewhat interchangeably.

It would be useful to understand how aspect angle varies within the HAIR region, to compare with the spectral characteristics of the HAIR echoes (see Sect. 3.4). From our observations and simple model it is difficult to determine the exact variation of aspect angle with range; however, we can estimate this, based on a simple argument. As discussed above, at ranges of $d_{\min }$ and beyond, where $\Delta \leq \Delta_{\max }$, the radar $\boldsymbol{k}$ is refracted to orthogonality with the magnetic field (rays A 

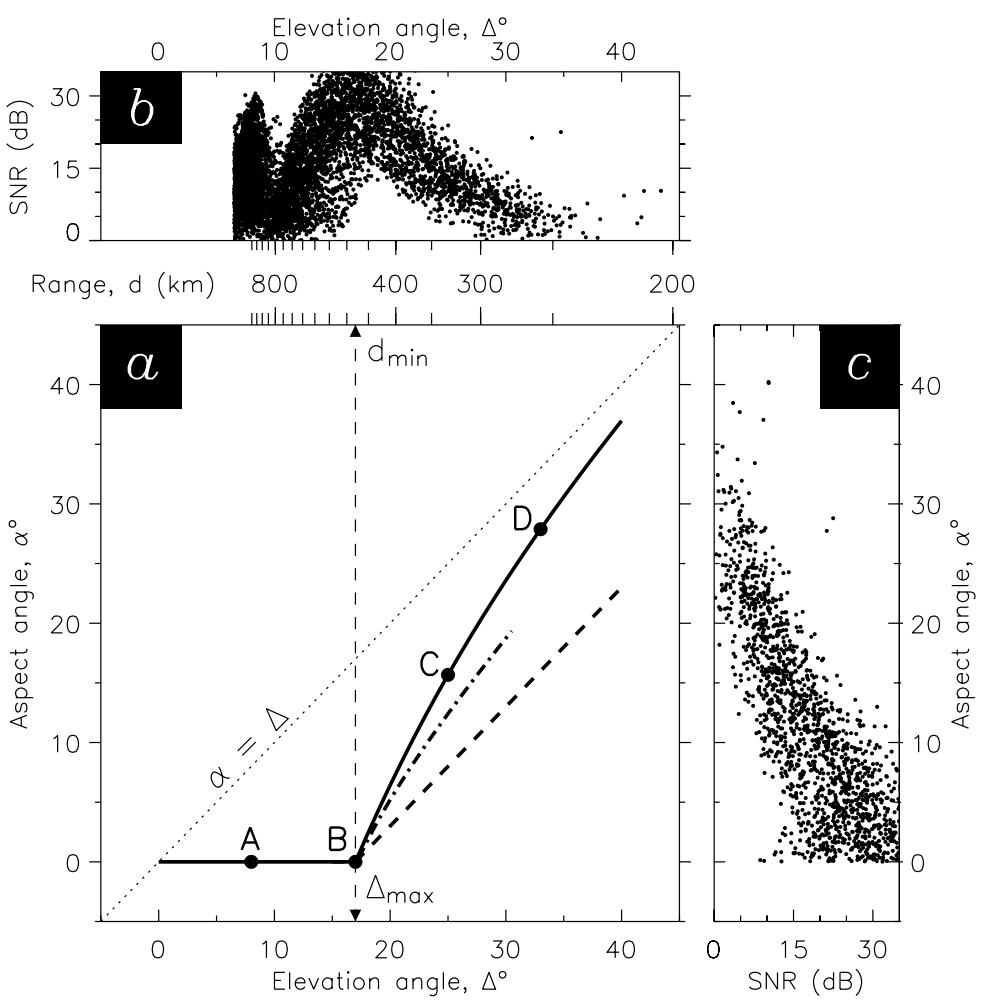

Fig. 6. (a) Estimates of the variation of aspect angle with increasing elevation angle, for $\Delta_{\max }=17^{\circ}$. Letters refer to rays in Fig. 5. See text for an explanation of the three curves. (b) The SNR observations from Fig. 2a, shown as a function of $\Delta$. (c) The SNR observations translated to a function of $\alpha$ using the solid curve of panel (a).

and B in Fig. 5) and $\alpha=0^{\circ}$. At ranges closer than $d_{\text {min }}$ the refraction of the radar signal in the ionosphere is insufficient to bring the radar $\boldsymbol{k}$ to orthogonality. Rays launched at elevation angles just above $\Delta_{\max }$ will be refracted almost to orthogonality $\left(\alpha \approx 0^{\circ}\right)$, whereas as $\Delta$ increases further rays experience less and less refraction (ray $\mathrm{C}$ ), until the angle between $\boldsymbol{B}$ and $\boldsymbol{k}$ is close to $\zeta$, in which case the aspect angle $\alpha \approx \Delta$ (ray D), i.e. for $\Delta \leq \Delta_{\max }$ the aspect angle $\alpha=0^{\circ}$, but $\alpha$ should increase rapidly from a value of $0^{\circ}$ to a value close to $\Delta$, as $\Delta$ increases above $\Delta_{\max }$. Several potential relationships between $\alpha$ and $\Delta$ are illustrated in Fig. 6a. The solid curve presents a best-guess relationship, assuming that the magnetic field is vertical; this curve shows an asymptotic approach of $\alpha$ to $\Delta$, as $\Delta$ increases; in the range shown this can be approximated as $\alpha \approx 2\left(\Delta-\Delta_{\max }\right)$. The dashed curve shows the minimum possible increase in $\alpha$ with $\Delta$, that is an increase of $1^{\circ}$ in $\alpha$ for every increase of $1^{\circ}$ in $\Delta$, or $\alpha=\Delta-\Delta_{\max }$. Finally, the dot-dashed curve shows the relationship found from a simple ray-tracing analysis, through a parabolic E-region, with $f o \mathrm{E}=3 \mathrm{MHz}$ and $f_{r}=10 \mathrm{MHz}$, assuming that the magnetic field is inclined at $5^{\circ}$ to the vertical; in this case, $\alpha \approx \frac{3}{2}\left(\Delta-\Delta_{\max }\right)$. These variations will be discussed further in Sect. 3.4.

A final point should be considered. If our assumptions about the propagation are correct, Fig. 5a suggests that regions of normal-echo backscatter should be accompanied by ground scatter at twice the range. No ground scatter is expected to be associated with the HAIR echoes, as these rays do not reflect in the E-region, and so there should be a cut- off in ground echoes at a range closer than $d_{\text {skip }} \approx 2 d_{\text {min }}$ (or $2 d_{\text {HAIR }}$ ) (Fig. 5c). It was the general association between E-region echoes and ground scatter that originally lead Milan and Lester (1998) to suggest that backscatter originated in regions of enhanced electron density, and many examples showing this association can be found. One particularly clear example, from 10 October 1997, is shown in Fig. 7. During this interval the radar was operating in myopic mode, though solely at a frequency of $10 \mathrm{MHz}$. Figure 7 a shows Doppler shift as a function of range and time from beam 12 of the radar. At nearer ranges a band of E-region scatter approaches the radar. Doppler shifts are predominantly positive as the radar is located pre-midnight in the eastward electrojet. After 17:10 UT reverse-Doppler shift HAIR echoes appear at the leading edge of the backscatter. In this example the power maximum appears approximately two range gates beyond the Doppler shift reversal, indicated by a dashed line; this is $d_{\text {HAIR }}\left(d_{\text {min }}\right)$. The variation of $d_{\text {HAIR }}$ with time suggests that not only is the region of elevated electron density moving equatorward, but that $f_{o} \mathrm{E}$ is also increasing slightly (see Fig. 4). Further from the radar, zero-Doppler shift echoes denote the observation of ground backscatter (indicated in grey), which also approaches the radar with time. Superimposed is a dot-dashed line showing the variation of the range

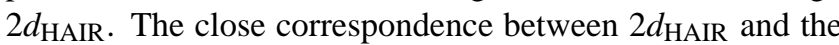
leading edge of the ground backscatter (the skip distance, $d_{\text {skip }}$ ) clearly supports our hypothesis. Figures $7 \mathrm{~b}$ and $\mathrm{c}$ indicate the relationship between $d_{\text {HAIR }}, 2 d_{\text {HAIR }}$, and ground backscatter in a single scan from this interval. In Fig. $7 \mathrm{~b}$ the 

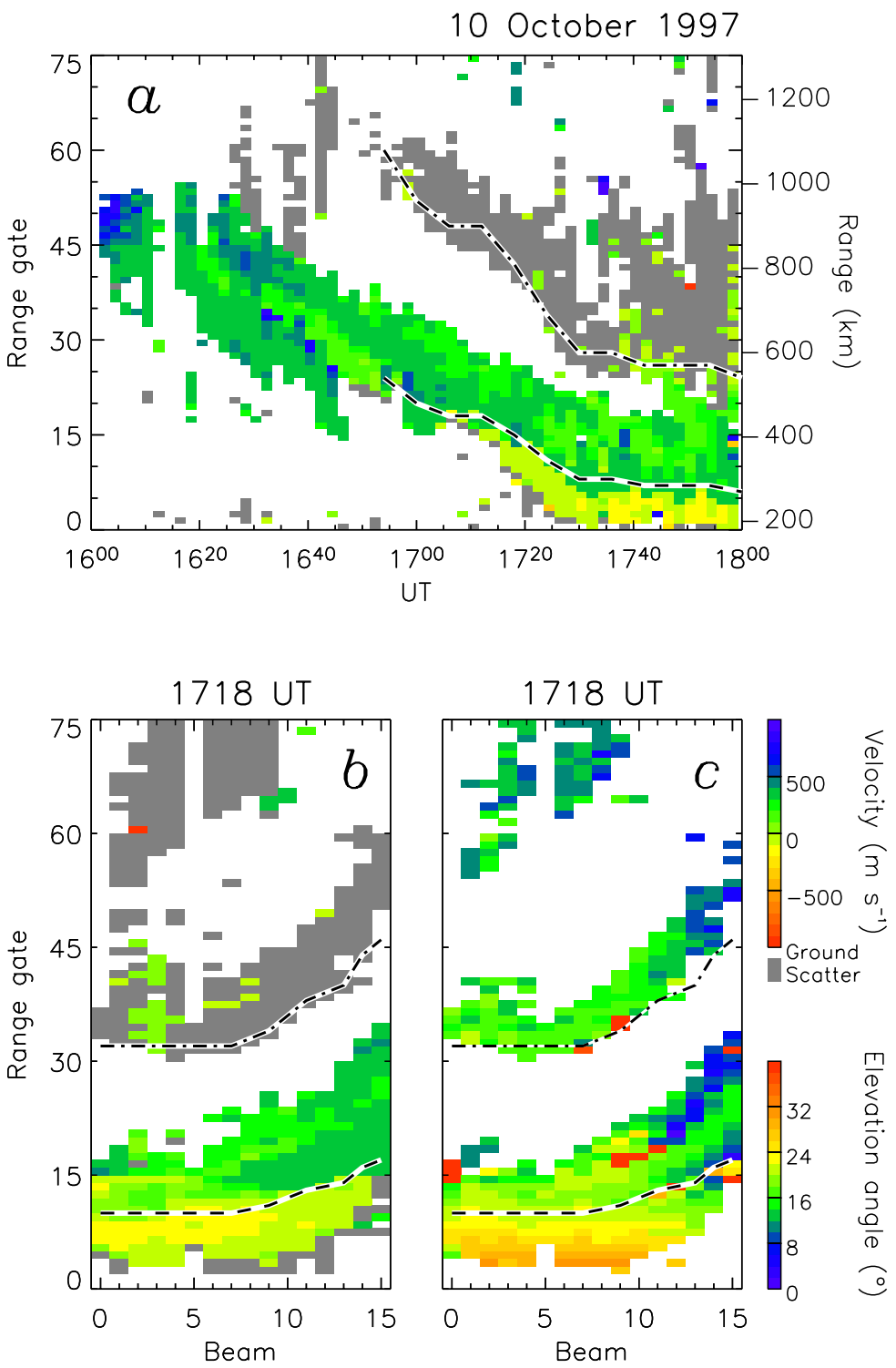

Fig. 7. (a) Range-time-Doppler shift plot for beam 12, 16:00-18:00 UT, 10 October 1997. The dashed line indicates the range to the nearest normal echoes, $d_{\text {HAIR }}$, and the dot-dashed curve the range $2 d_{\text {HAIR }}$ (expected to be close to $d_{\text {skip }}$ ). (b) Doppler shift and (c) elevation angle for the scan starting 17:18 UT.
Doppler shift reversal is clearly visible near $d_{\text {HAIR }}$. Figure 7c shows the simultaneous elevation angle measurements. This indicates that the ground scatter and the region of normal echoes (beyond the dashed line) have similar elevation angles, that is are sampled by the same rays. The HAIR echoes, however, have larger elevation angles than seen in the ground scatter, or in other words, the rays which sense these echoes are not reflected in the E-region and hence, do not give rise to ground backscatter. This again supports the picture presented in Figs. 5a and c.

To emphasize this point, Fig. 8 shows elevation angle, Doppler shift and SNR as a function of range for the scan shown in Figs. 7b and c. Near-zero Doppler shift echoes beyond range gate 32 , marked by a vertical dot-dashed line, are ground scatter echoes, and at nearer ranges E-region echoes are observed. The E-region echoes can be sub-divided into HAIR and normal echoes at range gate 15, based on their
Doppler shift and SNR characteristics. Normal echoes have elevation angles below $18^{\circ}$ (horizontal dashed line), as indeed do the ground echoes. As another check, lower and upper green dashed lines show the expected variation of $\Delta$ for ionospheric echoes originating from a virtual height of $120 \mathrm{~km}$, and ground echoes reflected at a virtual height of $120 \mathrm{~km}$, respectively. Satisfactory agreement is found with the observations.

In our original examples (Fig. 1) the picture at far ranges is somewhat complicated due to the presence of F-region scatter. However, near-zero Doppler shift ground scatter echoes are observed, especially in lower-numbered beams in the eastwards electrojet (20:00-20:30 UT, 14 December 2001), example $a$. At $10 \mathrm{MHz}$ these echoes are consistently observed at range gate 45 and beyond, though $d_{\text {skip }}$ is clearly a function of frequency (channel B). Figure 9 shows the frequency-variation of $d_{v e l}$ and $d_{\text {HAIR }}$ (the same variations 

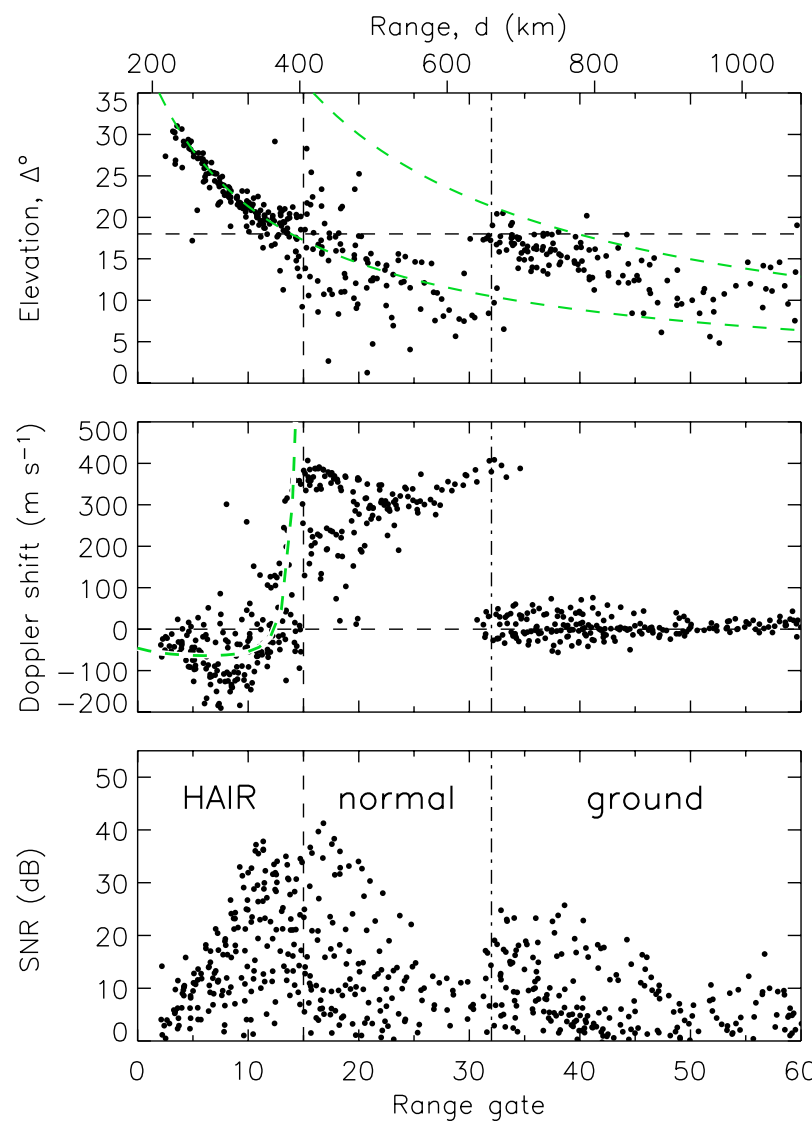

Fig. 8. Elevation angle, Doppler shift, and SNR as a function of range for the scan presented in Fig. 7. Vertical dashed and dotdashed lines indicate $d_{\text {HAIR }}$ and $d_{\text {skip. }}$. In the top panel, lower (upper) green dashed curve shows the expected variation of elevation angle for ionospheric (ground) echoes scattered (reflected) from a virtual height of $120 \mathrm{~km}$. In the middle panel, green dashed curve indicates the modeled variation of irregularity phase speed for central beams of the radar (see also Fig. 13).

as shown in Fig. 4), together with that of $d_{\text {skip. Superim- }}$ posed is the variation of $2 d_{\text {HAIR }}$, which should be close to $d_{\text {skip. Although }} d_{\text {HAIR }}$ is only known for the lower frequency bands, we find consistency with our simple model. For completeness the variation of $2 d_{v e l}$ is also shown.

The picture we have presented above is clearly idealized. The Earth is not flat, the magnetic field is not vertical, and $f o \mathrm{E}$ is not uniform throughout the electrojet region, and these factors will affect the predictions made above. For instance, it can be envisaged that because the magnetic field is not exactly vertical, then the point of orthogonality does not occur at the exact reflection point (cf. Fig. 5a) and so ground backscatter should be observed at ranges somewhat further than $2 d_{\text {HAIR. }}$. To properly model the variation of $d_{\min }$ with foE and radar frequency (Fig. 4), or the location of ground scatter relative to $d_{\min }$ (Figs. 7 and 9), it is necessary to ray-trace through model electron density profiles. This introduces many variables into the melting pot, including the variation of electron density with altitude, the variation of

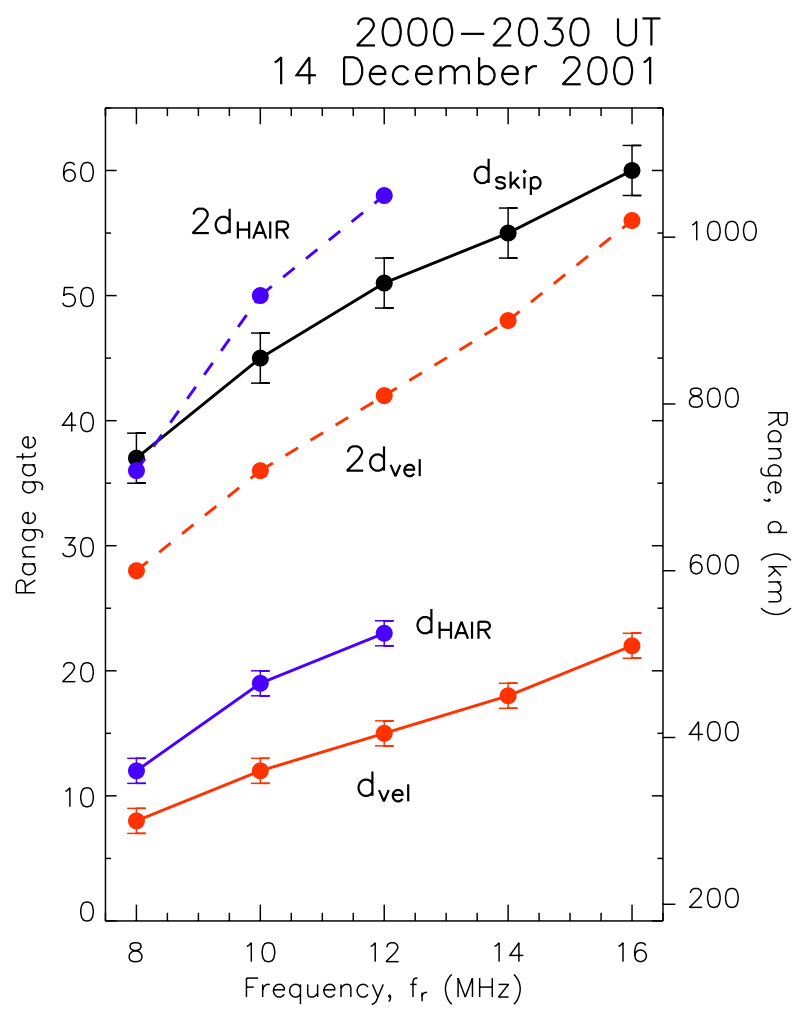

Fig. 9. The variation of $d_{v e l}, d_{\text {HAIR }}$ and $d_{\text {skip }}$ as a function of frequency for the example from Fig. 1a. The variations of $2 d_{v e l}$ and $2 d_{\text {HAIR }}$ are also shown.

foE with latitude, and the tilt of the magnetic field, which depends on the radar look-direction. A detailed discussion of ray-tracing results is beyond the scope of the present paper, though initial findings broadly support our much simplified analysis. For instance, as mentioned above, the aspect angle does appear to increase rapidly once orthogonality can no longer be achieved. Ray-tracing also shows that due to the tilt of the magnetic field, orthogonality can be achieved even without the reflection criterion being met, or in other words, that ground echoes are not necessarily always associated with normal echoes, unless foE is relatively high. In addition, when ground echoes are present, and if $f o \mathrm{E}$ is assumed uniform, then $d_{\text {skip }}$ is found to be $\beta d_{\text {min }}$, where $\beta$ can vary between 2 and 2.4. On the other hand, if $f_{o} \mathrm{E}$ is assumed to increase with latitude in the equatorward portion of the electrojet (e.g. Whalen, 1983, and see Fig. 5a), then the ionosphere acts as a tilted reflecting surface, tending to bring $\beta$ back to values near 2 or less.

\subsection{HAIR Doppler shift characteristics}

Now we turn to an investigation of the Doppler shift characteristics of the HAIR echoes, and especially the azimuthal dependence of the Doppler shift. In this study we measure azimuthal angles (or L-shell angles) anticlockwise from magnetic east. E-region echoes are generally found to be Doppler-shifted along the direction of electron $\boldsymbol{E} \times \boldsymbol{B}$ drift in 

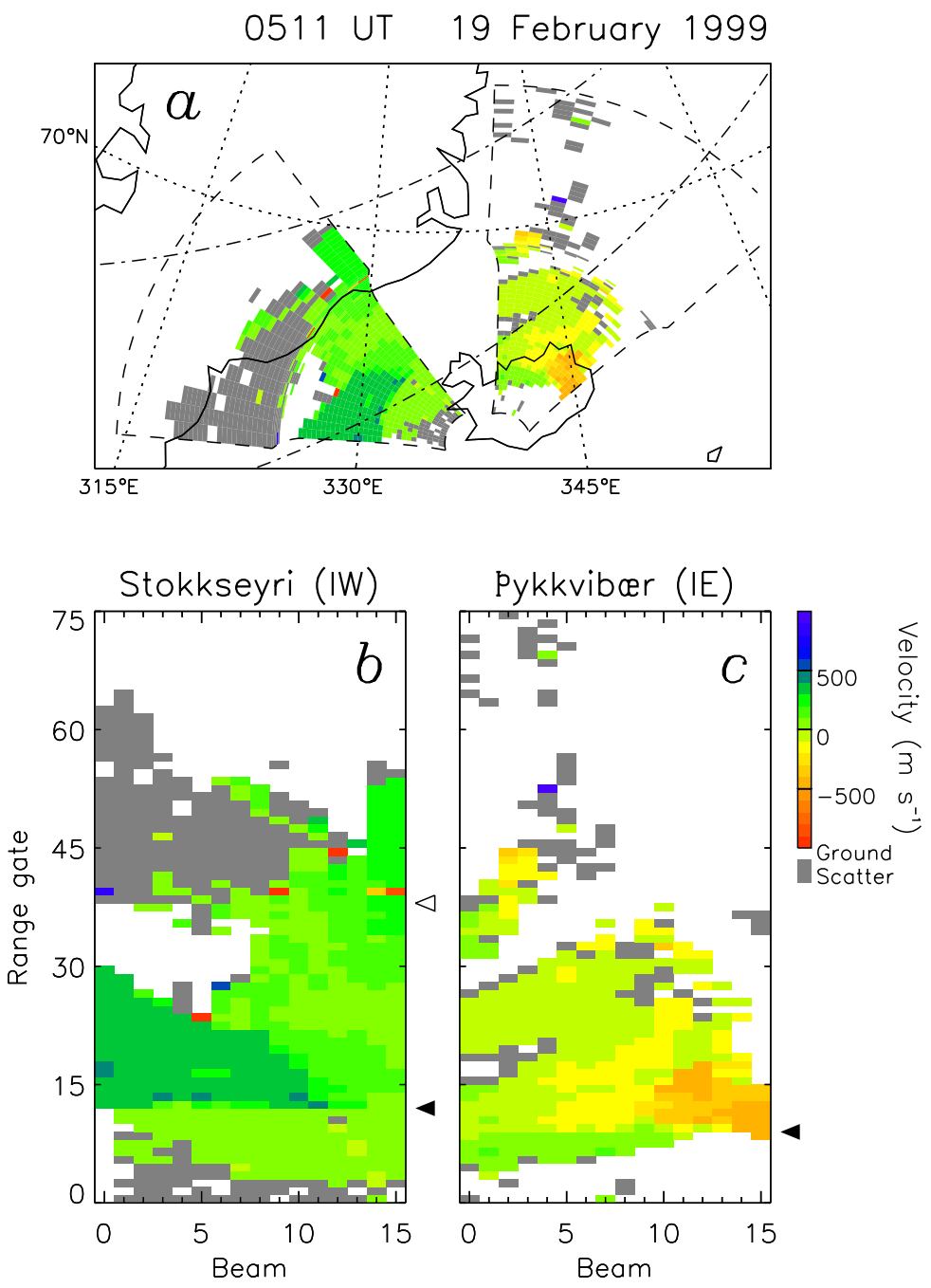

Fig. 10. (a) IW and IE fields of view from 05:11 UT, 19 February 1999, presented in a geographic coordinate frame. Superimposed as dot-dashed lines are the poleward and equatorward edges of the statistical auroral oval. (b) and (c) The Doppler shift measurements indicating $d_{v e l}$ (filled triangles) and, in the case of IW, $d_{\text {skip }}$ (unfilled triangle).

the electrojet regions, such that our eastward-pointing radar observes mainly positive velocities in the eastward electrojet and negative velocities in the westward electrojet. If the Doppler shift of echoes is directly controlled by the electric field, and this is assumed to be spatially uniform throughout the region of backscatter, then a cosine dependence is expected between Doppler shift and azimuthal look direction (e.g. Villain et al., 1987, 1990; Milan et al., 2001, 2003a), such that

$-V_{\text {los }}=V_{\text {flow }} \cos \left(\phi-\phi_{\text {flow }}\right)$,

where $V_{l o s}$ is the line-of-sight Doppler shift (made negative as Doppler shift is conventionally positive towards the radar), $V_{\text {flow }}$ is the background drift velocity, $\phi_{\text {flow }}$ is the azimuthal direction of the drift $\left(\phi_{\text {flow }}=0^{\circ}\right.$ would represent Lshell aligned electron drift in the westward electrojet), and $\phi$ is the local azimuthal look-direction of the radar beam. In general E-region echoes do not follow exactly this relationship due to threshold effects and sound-speed limiting; as mentioned before echoes can comprise two Doppler shift components with high and low velocities, respectively
(TWOPEE echoes), each responding to the electric field in different ways. However, it is clear that at many times this cosine dependence provides a useful context for understanding the variation of Doppler shift within the radar field-ofview.

An individual SuperDARN radar has an azimuth coverage of $52^{\circ}$. Two nearby radars pointing in different directions, such as the Iceland East and West radars (IE and IW) shown in Fig. 10a, can effectively be thought of as a single radar with a much greater range of azimuthal look-directions, in this case close to $150^{\circ}$. Milan et al. (2000) used this pair of radars to show that in the electrojets if one radar observed predominantly negative velocities, the other measured positive velocities, and vice versa, consistent with the dominant direction of irregularity drift being westward or eastward. Here we exploit the extended azimuthal coverage of the radar pair to investigate the Doppler shift characteristics of the HAIR echoes.

Figure 10a presents observations of Doppler shift from IE and IW at 05:11 UT, 19 February 1999, when both were operating in myopic mode, that is $15 \mathrm{~km}$ range gates. At this 
time IE and IW were operating at 10 and $12 \mathrm{MHz}$, respectively. The radars are located in the westward electrojet, and as a consequence, Doppler shifts are primarily positive and negative in the IW and IE fields-of-view. In addition, HAIR echoes are present at near ranges in both fields-of-view (Fig. $10 \mathrm{~b}-\mathrm{c}$ ), $d_{v e l}$ being indicated by filled triangles. In the case of IE the Doppler shifts of the HAIR echoes are as described above, being low and positive in the westward electrojet, oppositely directed to the normal scatter. In the IW observations there is again a clear demarcation between HAIR and normal echoes, except that here the HAIR Doppler shifts are of the same sense as the normal echoes, i.e. both positive. Figure 11 shows this more clearly, presented in a similar format to Fig. 8, the upper and lower panels corresponding to IW and IE, respectively. The boundary between HAIR and normal echoes is clear at gates 14 and 11 for the two radars, respectively $\left(d_{\text {HAIR }}=390\right.$ and $345 \mathrm{~km}$, indicated by vertical dashed lines), confirmed not only by the Doppler shifts, but also supported by the characteristic variation of SNR with range. For IE this marks a transition from negative-Doppler shift HAIR echoes to positive-Doppler shift normal echoes. In the case of IW, both HAIR and normal echoes are positively Doppler shifted. Indeed, this is generally found to be the case, that is in IE observations HAIR and normal echoes have velocities of opposite sense, but are of the same sense in IW observations, regardless of whether the radars are located in the eastward or westward electrojet.

As an aside, we note that IW observes ground scatter at further ranges. The open triangle in Fig. 10 marks $d_{\text {skip }}$ $(750 \mathrm{~km})$, as does the vertical dot-dashed line in Fig. 11. As before, $d_{\text {skip }} \approx 2 d_{\text {HAIR }}$. Furthermore, $d_{\text {HAIR }}$ differs slightly for the two radars as a consequence of their different operating frequencies. These values of $d_{\text {HAIR }}$ have been shown with their corresponding values of $f_{r}$ in Fig. 4 (green dots), showing consistency with a value of $f o \mathrm{E}$ between 3 and 3.5 MHz.

In Fig. 12a we investigate the azimuthal variation of Doppler shift from the radar scans shown in Fig. 10. The IW field-of-view covers the azimuth range $100^{\circ}<\phi<160^{\circ}$, with IE values of $\mathrm{L}$-shell angle falling between $10^{\circ}<\phi<65^{\circ}$. Normal echoes are indicated in black, HAIR echoes in red. Two main points are clear. For IW both normal and HAIR echoes have positive Doppler shifts, whereas they are oppositely directed for IE. Also, two blue superimposed cosine curves $\left(V_{\text {flow }}=200,450 \mathrm{~m} \mathrm{~s}^{-1}, \phi_{\text {flow }}=0^{\circ}\right)$ show that most normal echoes can be satisfactorily explained in terms of eastwards drift. However, the HAIR echoes are more consistent with the green superimposed cosine curve $\left(V_{\text {flow }}=70 \mathrm{~m} \mathrm{~s}^{-1}, \phi_{\text {flow }}=-90^{\circ}\right)$, indicating slower equatorward flow. Figure $12 \mathrm{~b}$ shows observations from some four hours earlier in the day (still in the westward electrojet), indicating that the same general relationship holds. Figures 12c and $d$ present measurements made on the 25 September 2000 when both radars were in the eastward electrojet. Here the situation is reversed, with normal echoes being consistent with westward flow and HAIR echoes with poleward flow. In both cases the superimposed HAIR curve has

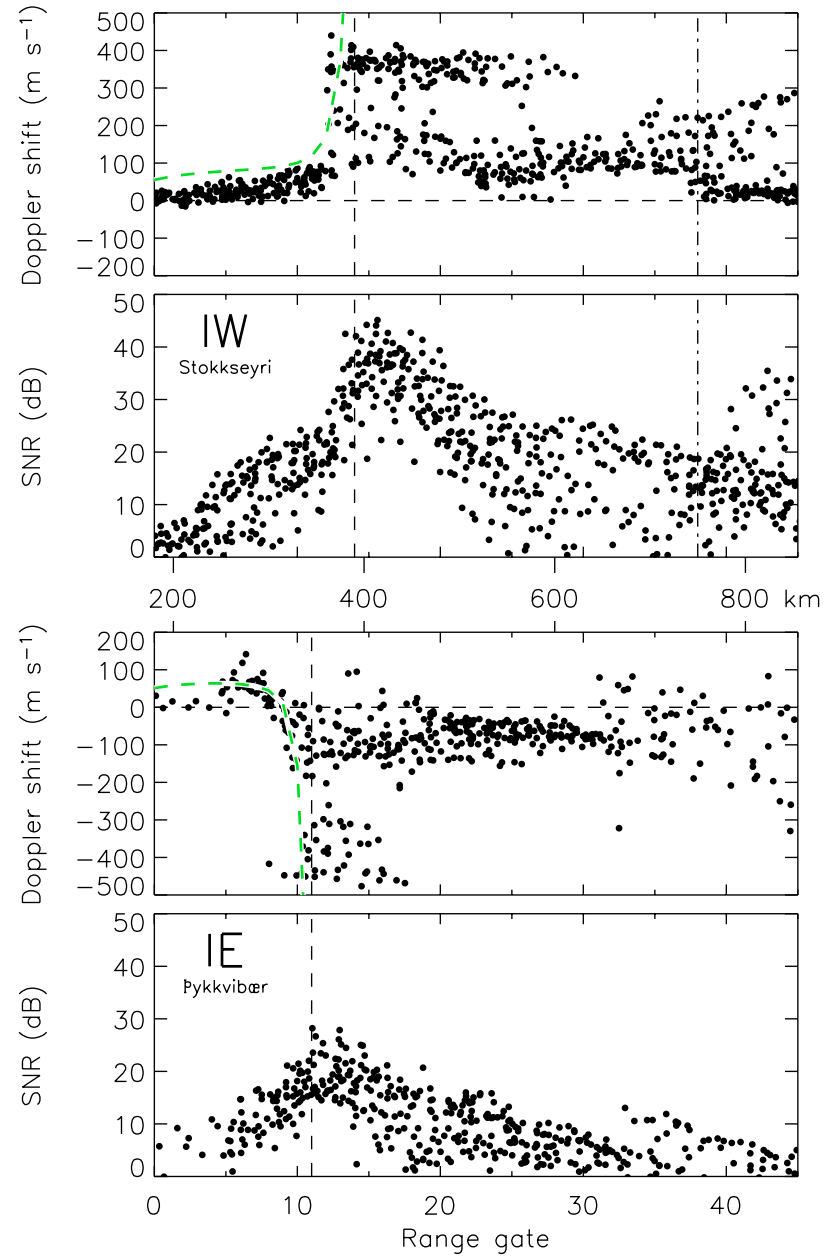

Fig. 11. Doppler shift and SNR variation as a function of range for the IW and IE observations of Fig. 10. Vertical dashed and dotdashed lines indicate $d_{\text {HAIR }}$ and $d_{\text {skip }}$, respectively. Green dashed curves indicate the modeled variation of irregularity phase speed for central beams of the two radars (see also Fig. 13).

$V_{\text {flow }}=130 \mathrm{~m} \mathrm{~s}^{-1}$ and $\phi_{\text {flow }} \approx 90^{\circ}$, though in Fig. $12 \mathrm{~d}$ a constant offset of $80 \mathrm{~m} \mathrm{~s}^{-1}$ has been added to achieve a good fit with the data. The occurrence of poleward- and equatorwarddirected HAIR flow in the eastward and westward electrojets, respectively, appears to hold quite generally in all examples found to date. This suggests that while normal echoes appear to drift in a direction consistent with $\boldsymbol{E} \times \boldsymbol{B}$, the HAIR echoes are more consistent with drift in the direction of $\boldsymbol{E}$, which is directed poleward and equatorward in the eastward and westward electrojets, respectively. In addition, while normal echoes can achieve Doppler shifts of several hundred $\mathrm{m} \mathrm{s}^{-1}$ $\left(800 \mathrm{~m} \mathrm{~s}^{-1}\right.$ appears to be an approximate upper limit, Milan et al., 2003a), HAIR echoes are rarely observed with Doppler shifts in excess of $100-150 \mathrm{~m} \mathrm{~s}^{-1}$.

These Doppler shift characteristics can be understood in terms of the linear dispersion relation for plasma waves in the auroral electrojets. In the rest frame the oscillation frequency can be expressed as (e.g. Fejer and Kelley, 1980; Fejer and Providakes, 1987; Haldoupis, 1989): 

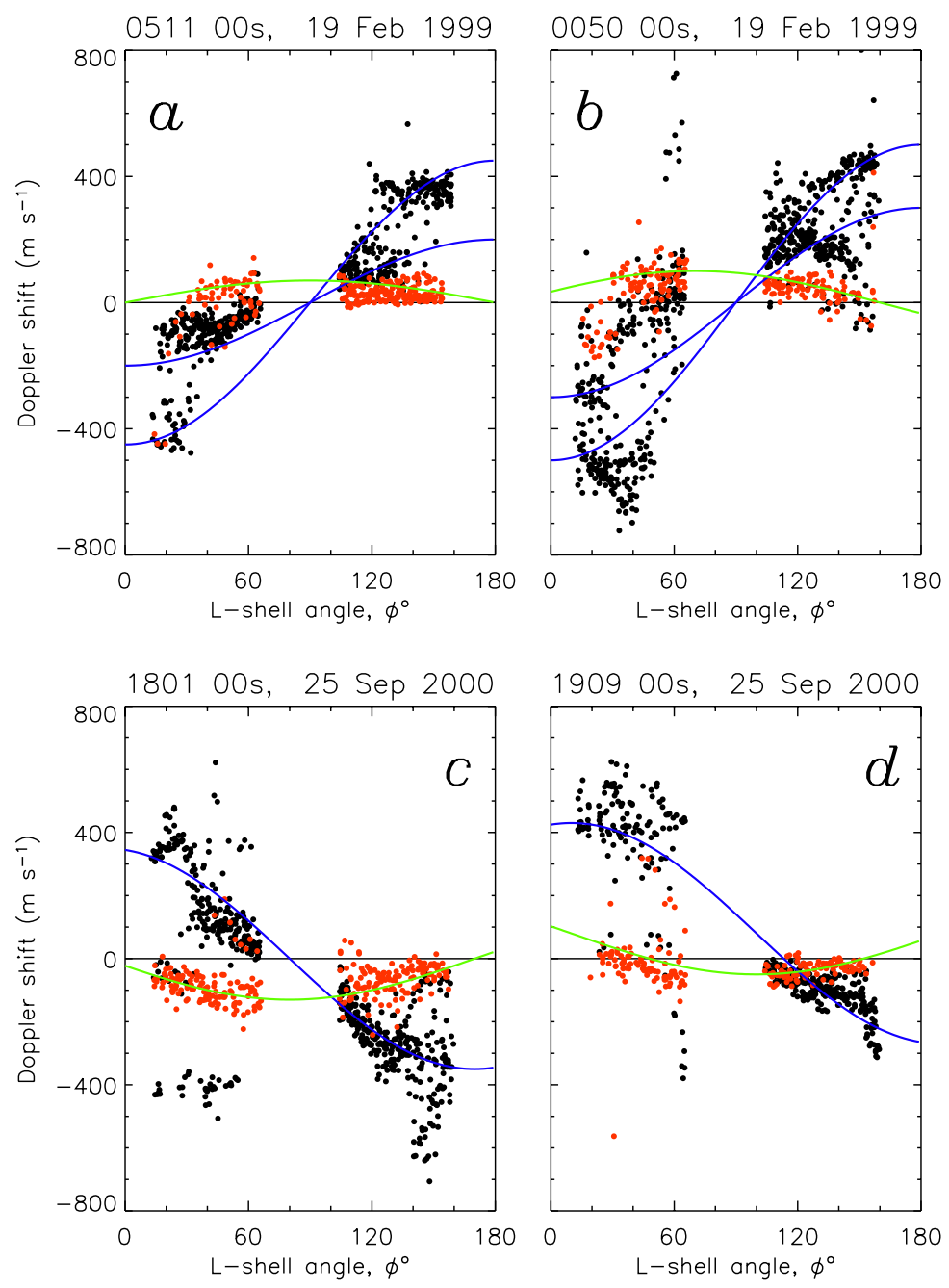

Fig. 12. Doppler shift as a function of L-shell angle for two examples from the westward electrojet (a) and (b) and two from the eastward electrojet (c) and (d). Normal echoes are indicated in black, HAIR echoes in red. Superimposed blue curves show rough expectations for eastward or westward flow, whereas the green curves show poleward or equatorward flow.

$\omega_{r}=\frac{1}{1+\Psi} \boldsymbol{k} \cdot \boldsymbol{V}_{\boldsymbol{e}}+\frac{\Psi}{1+\Psi} \boldsymbol{k} \cdot \boldsymbol{V}_{\boldsymbol{i}}$,

where

$\Psi=\frac{v_{e} v_{i}}{\Omega_{e} \Omega_{i}}\left(\cos ^{2} \alpha+\frac{\Omega_{e}^{2}}{v_{e}^{2}} \sin ^{2} \alpha\right)$,

$\boldsymbol{k}$ is the wave vector, $\boldsymbol{V}_{e}$ and $\boldsymbol{V}_{i}$ are the electron and ion drift velocities given by

$\boldsymbol{V}_{\boldsymbol{e}}=\frac{\Omega_{e}^{2}}{v_{e}^{2}+\Omega_{e}^{2}} \frac{\boldsymbol{E} \times \boldsymbol{B}}{B^{2}}-\frac{v_{e} \Omega_{e}}{v_{e}^{2}+\Omega_{e}^{2}} \frac{\boldsymbol{E}}{B}$

$\boldsymbol{V}_{\boldsymbol{i}}=\frac{\Omega_{i}^{2}}{v_{i}^{2}+\Omega_{i}^{2}} \frac{\boldsymbol{E} \times \boldsymbol{B}}{B^{2}}+\frac{v_{i} \Omega_{i}}{v_{i}^{2}+\Omega_{i}^{2}} \frac{\boldsymbol{E}}{B}$,

and $v_{e}\left(v_{i}\right)$ is the electron (ion) collision frequency, $\Omega_{e}\left(\Omega_{i}\right)$ is the electron (ion) gyrofrequency, and $\alpha$ is the aspect angle as before. In the following calculations we use the values of collision and gyrofrequencies given by Fejer and
Providakes (1987) which are appropriate for an altitude of $105 \mathrm{~km}: v_{e}=4 \times 10^{4} \mathrm{~s}^{-1}, v_{i}=2.5 \times 10^{3} \mathrm{~s}^{-1}, \Omega_{e}=10^{7} \mathrm{~s}^{-1}$ and $\Omega_{i}=180 \mathrm{~s}^{-1}$. Then Eqs. (10) and (11) show that electrons drift in the $\boldsymbol{E} \times \boldsymbol{B}$ direction with a speed very close to $E / B$, and ions drift predominantly in the direction of $\boldsymbol{E}$ at a speed of less than $10 \%$ of $E / B$. For field-aligned irregularities, $\alpha=0^{\circ}$ so $\Psi$ is small ( $\Psi \approx 0.06$ ), and consequently, the first term on the right-hand side of Eq. (8) dominates. In this case, the phase speed of irregularities, and hence, the Doppler shift determined by the radar $V_{l o s}=\omega_{r} / k$, is expected to be close to the line-of-sight component of the electron drift velocity $\boldsymbol{V}_{e}$, with electrons moving in the $\boldsymbol{E} \times \boldsymbol{B}$ direction. This is broadly consistent with our observations of normal electrojet echoes. On the other hand, as aspect angle increases, $\Psi$ rapidly becomes very large $\left(\Psi \approx 1.1\right.$ for $\alpha=1^{\circ}, \Psi \approx 26$ for $\alpha=5^{\circ}$ ). Then the second term on the right-hand side of Eq. (8) becomes increasingly significant and dominates for $\alpha>1^{\circ}$. For sufficiently high $\alpha$, such that the $\boldsymbol{V}_{e}$ term can be neglected, Eq. (8) reduces to

$\omega_{r} / k \approx V_{i} \cos \theta \cos \alpha$, 
where $\theta$ is the angle between the radar azimuthal lookdirection and the ion drift. Consequently, at high aspect angles the phase speed is close to the line-of-sight component of the ion drift, which is directed along $\boldsymbol{E}$ with a magnitude approximately one-tenth of the electron drift speed. At the very highest aspect angles the velocity again drops as the radar beam passes increasingly vertically through the ionosphere, expressed by the $\cos \alpha$ dependence in Eq. (12). Both a depressed phase speed and a $90^{\circ}$ rotation in flow direction with respect to normal echoes are consistent with our observed HAIR echo characteristics.

As discussed in Sect. 3.2, aspect angle is expected to vary with range in the HAIR. Equations (8) to (12) can be used to predict the variation of Doppler shift with aspect angle, and hence, range. Figure 13a shows $\omega_{r} / k$ as a function of $\alpha$ for two radar look-directions, $\phi=45^{\circ}$ (solid curve) and $135^{\circ}$ (dashed curve), corresponding approximately to central beams of IE and IW, respectively. Here we have assumed the electron flow speed $E / B=1.5 \mathrm{~km} \mathrm{~s}^{-1}$ with L-shell angle $\phi_{\text {flow }}=0^{\circ}$ (appropriate for the westward electrojet case shown in Figs. 10 and 11). Figure 13a shows that for fieldaligned irregularities $\omega_{r} / k \approx \pm 1 \mathrm{~km} \mathrm{~s}^{-1}$ (though this would in general be limited, for instance, to approximately the ionacoustic speed in the case of type I spectra) but decreases in magnitude rapidly for $\alpha>0^{\circ}$, towards $\sim 100 \mathrm{~m} \mathrm{~s}^{-1}$ for both IE and IW. The Doppler shift remains positive for all $\alpha$ for the IW radar. In the case of IE, however, the Doppler shift swaps sign for $\alpha \geq 4^{\circ}$, to give rise to the characteristic reverse-Doppler signature seen with this radar. As the change in Doppler shift sign does not occur for $\alpha<4^{\circ}$, this explains why SNR always appears to maximize a few range gates beyond the Doppler shift reversal, or in other words, why $d_{\text {vel }}<d_{\text {HAIR. }}$. Figure $13 \mathrm{~b}$ presents the same curves as Fig. 13a, though concentrating on lower phase speeds and showing the full possible range of $\alpha$, with four additional curves for radar look-directions $\pm 25^{\circ}$ away from the central beams, that is the extremes of the fields-of-view. This indicates that at high aspect angles there is a look-direction variation in the phase speed, which maximizes in the ion drift direction. However, the aspect angle at which the velocity reversal occurs (in the case of the IE radar) does not vary significantly with look-direction. From Fig. 13a it is possible to estimate $\omega_{r} / k$ for central beams as a function of range (using the relationships presented in Fig. 6), which has been superimposed on Fig. 11 (green dashed curves), and good qualitative agreement is found. A similar curve has been superimposed on Fig. 8, this time appropriate for the eastward electrojet; again, good agreement is achieved.

Although the Doppler shift behaviour of the HAIR echoes can be explained by simple linear theory, the actual growth of high-aspect angle irregularities cannot. The growth rate provided by Fejer and Providakes (1987) suggests that the threshold drift velocity necessary for the generation of electrojet waves increases sharply for $\alpha>0^{\circ}$, such that the threshold for waves with $\alpha>3^{\circ}$ is unlikely ever to be achieved. However, more recently, Robinson and Schlegel (2000) have proposed a mechanism whereby electron collisional heating
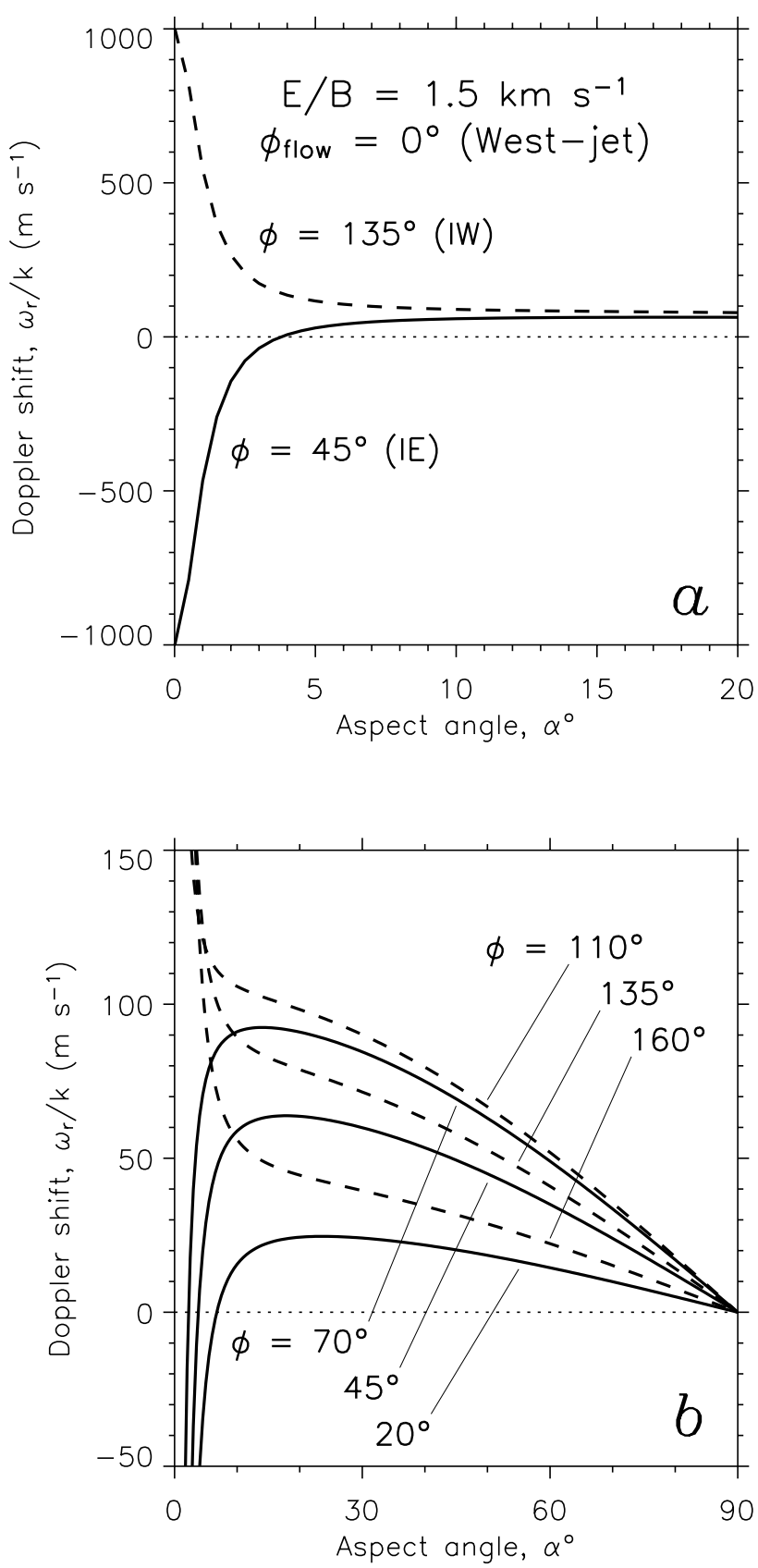

Fig. 13. (a) Modeled irregularity phase speed (Doppler shift) as a function of aspect angle for central beams of the IW and IE radars (see text for details). (b) Similar to (a), though concentrating on lower speeds and showing the full range of $\alpha$. Curves are also shown for the edges of the fields-of-view.

in the presence of modest field-aligned electron drifts (i.e. precipitation) can give rise to irregularities inclined at almost any angle to the magnetic field. Indeed, this mechanism was invoked by Robinson and Schlegel (2000) to explain backscatter observed by a $50 \mathrm{MHz}$ radar with beams pointing almost parallel to the magnetic field, i.e. from irregularities with $\alpha \approx 90^{\circ}$. The phase speed of such irregularities is predicted by Robinson and Schlegel (2000) to be very low 


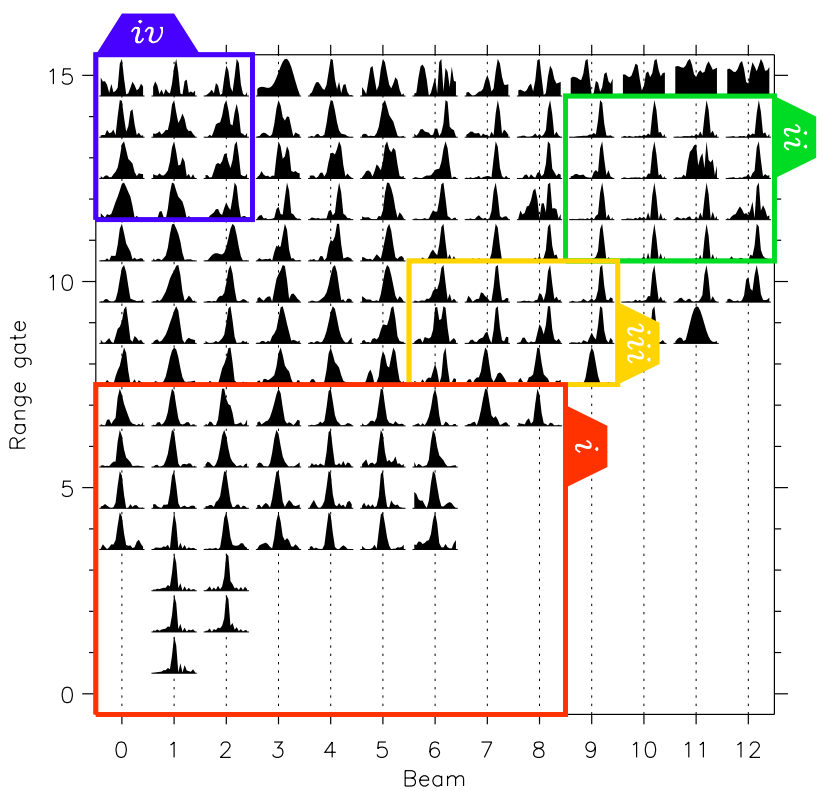

Fig. 14. Self-normalized Doppler spectra from the $8 \mathrm{MHz}$ observations of Fig. 1a. Spectra are shown for the Doppler shift range -1500 to $1500 \mathrm{~m} \mathrm{~s}^{-1}$. Boxed regions are described in the text.

indeed, of the order of a few $\mathrm{m} \mathrm{s}^{-1}$, though it is assumed in their theory that the ions are stationary with respect to the neutrals; inclusion of a relative ion drift allows us to regain Eq. (12). This mechanism is particularly suited to explaining the generation of the high-aspect angle waves associated with our HAIR observations, as it is thought to be most effective at long wavelengths, that is $10 \mathrm{~s}$ of metres, close to the operational wavelengths of HF radars. In addition, we know that the field-aligned electron drifts required by this instability mechanism are present, as this is the precipitation that gives rise to the region of electron density and conductivity enhancement (the electrojet) from which the radars scatter.

\subsection{Spectral width and power characteristics}

Having described the Doppler shift characteristics of HAIR spectra in some detail we now return to a discussion of the spectral width and power observations associated with these echoes. Thus far we have used the power, Doppler shift, and spectral width estimates provided by the standard SuperDARN ACF fitting technique. While these are a useful starting point, a major caveat must be emphasized. As discussed by Milan et al. (2003a), E-region echoes are often an amalgam of two or more spectral components, indicating that scatter from several irregularity types can occur within an individual radar range cell. These irregularity types may coexist within the ionosphere, be horizontally stratified, or may occur in regions which lie adjacent to each other with spatial scales smaller than $15 \mathrm{~km}$. When two or more spectral peaks co-exist the fitting technique yields a power that corresponds to the total echo power, a phase speed which corresponds to the Doppler shift of the strongest peak, and a spectral width which is more an indication of the separation of the peaks than the full width half maximum (FWHM) of either of the peaks. For this reason care must be exercised in interpretation of these values in terms of plasma-physical processes. As a consequence, we now examine the spectral shapes observed.

We concentrate on the spectral measurements made at $8 \mathrm{MHz}$ during the scan starting 20:01 UT on 14 December 2001 (Fig. 1), but note here that these are typical of all our observations, irrespective of radar frequency. Figure 14 shows the echo spectra for gates 0 to 15 in beams 0 to 12 from this scan. The spectra are self-normalized and depict the Doppler shift range $-1500 \mathrm{~m} \mathrm{~s}^{-1}$ to $1500 \mathrm{~m} \mathrm{~s}^{-1}$. Some regions of the field-of-view have been highlighted to aid discussion, numbered (i) to (iv) for description below.

Region (i) covers those echoes which we have identified as HAIR. These echoes have low negative Doppler shifts, though are broad and straddle the zero Doppler shift position. Typical Doppler shifts are $-50 \mathrm{~m} \mathrm{~s}^{-1}$, with FWHM of several $100 \mathrm{~m} \mathrm{~s}^{-1}$. There is an indication that the echoes increase in width with increasing range, which suggests that echoes are somewhat narrower at higher aspect angles (see Sect. 3.2 for a discussion of the variation of aspect angle with range). This increase in HAIR spectral width with range is also evident in Fig. 2.

Region (ii) indicates singly-peaked normal electrojet echoes. These have positive Doppler shifts, as expected for the sense of electron flow in the westward electrojet, with magnitudes of close to $700 \mathrm{~m} \mathrm{~s}^{-1}$ in these cases. The spectral peaks are typically $200-300 \mathrm{~m} \mathrm{~s}^{-1}$ wide. Region (iii) shows an area of overlap between regions (i) and (ii), that is in which two spectral peaks are present, one negatively and the other positively Doppler-shifted. This indicates that there is an overlap of perhaps 2 or 3 range gates between HAIR and normal echoes, as was discussed in Sect. 3.1. We believe that in this region both high- and low-aspect angles are sampled by rays with slightly differing elevation angles.

Finally, region (iv) again shows echoes with two spectral components, but in this case both positively Doppler-shifted, that is both shifted in the direction determined by the electron drift, but by differing amounts. The high Doppler shift peaks are similar to those in region (ii), and the low Doppler shift peaks are of a similar width. These are the TWOPEE echoes described by Milan et al. (2003a, b), mentioned previously. Milan et al. (2003b) suggested that the higher Doppler shift approximately corresponds to the line-of-sight component of the electron drift, and the lower Doppler shift is perhaps 10\% of this. It is interesting to note that this speed depression factor is similar to that of the HAIR echoes, but that the direction of the shift is not the same.

We now turn to a discussion of the power measurements, and attempt to relate these to the variation with aspect angle, that is the aspect sensitivity. Figure 6a shows estimates of the variation of $\alpha$ with range. As aspect angle increases the backscatter power of HAIR echoes might be expected to decrease sharply, and indeed at near ranges backscatter power falls as range decreases (Fig. 2), in other words, as 

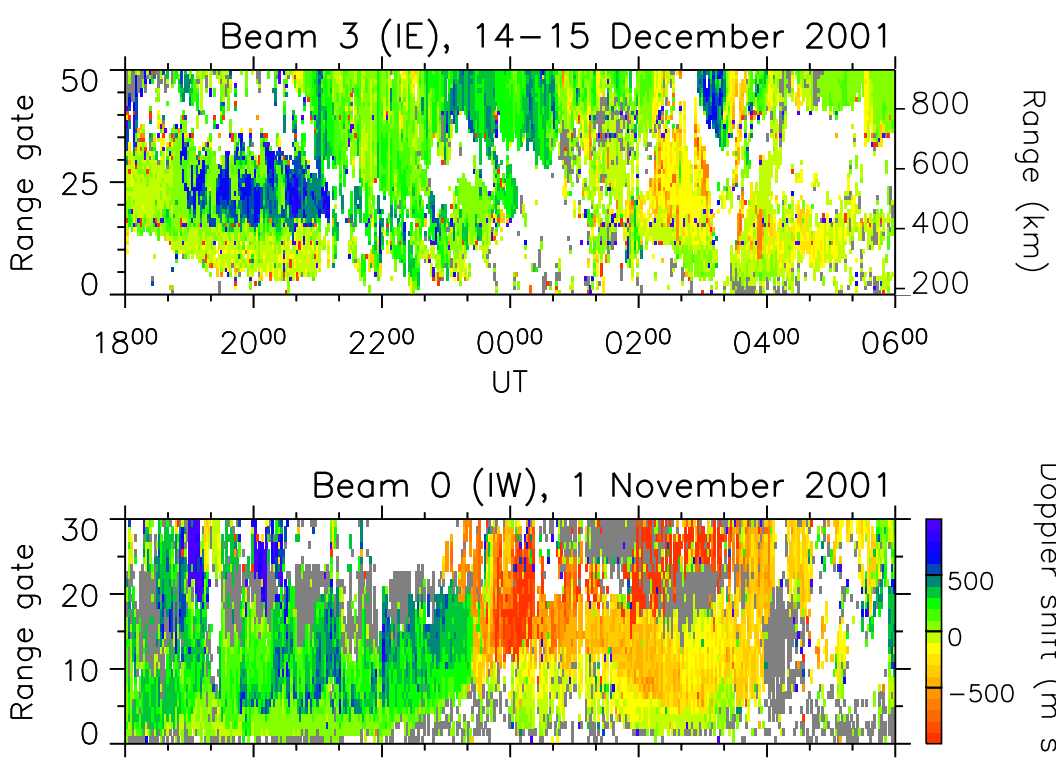

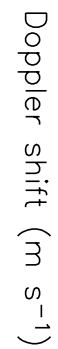

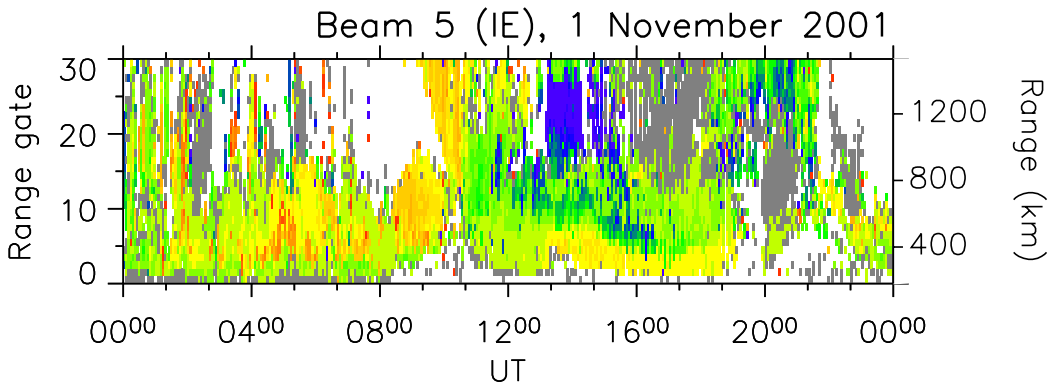

Fig. 15. Range-time-Doppler shift plots for the IE radar from 18:00-06:00 UT, 14-15 December 2001, and the IW and IE radars from 00:00-24:00 UT, 1 November 2001, demonstrating the ubiquity of HAIR echoes. (Note that in the lower two panels, the range resolution is $45 \mathrm{~km}$, instead of $15 \mathrm{~km}$.)
$\Delta$ increases, at a rate close to $-1 \mathrm{~dB}$ for each degree of $\Delta$. Figure $6 \mathrm{~b}$ shows the variation of SNR (taken from Fig. 2a) with range $d$, or equally $\Delta$, the two being related through Eq. (3) for a fixed virtual height. The curves of Fig. 6 a then allow the relationship between SNR and $\alpha$ to be estimated. The solid curve gives rise to Fig. 6c, which shows that SNR falls by approximately $-0.5 \mathrm{~dB}$ for every degree of $\alpha$. This also suggests that scatter is seen at aspect angles as high as $30^{\circ}$. Depending on the assumptions made, that is which of the curves of Fig. 6a is chosen, the aspect sensitivity can be estimated to be between $0.5-1 \mathrm{~dB} \mathrm{deg}^{-1}$. Although a decline in power with increasing aspect angle is expected, this rate is much slower than the aspect sensitivity determined at VHF and UHF frequencies (e.g. Moorcroft, 1985; Nielsen, 1988; and references therein), where values of $10 \mathrm{~dB} \mathrm{deg}^{-1}$ are common.

\subsection{HAIR occurrence}

Once the characteristics of HAIR echoes are recognized it becomes clear that high-aspect angle scatter is a common feature of HF radar observations. The upper panel of Fig. 15 shows a range-time-Doppler shift plot from beam 3 of channel A $(10 \mathrm{MHz})$ of the IE radar for 18:00-06:00 UT, 14-15 December 2001, the interval containing the two examples shown in Fig. 1. A clear Doppler shift reversal can be seen near range gate 13 between 18:30 and 21:00 UT, in the eastward electrojet, indicating that the HAIR was present here for some $2.5 \mathrm{~h}$. HAIR echoes were less common during the westward electrojet portion of the night, though can clearly be seen between 02:30 and 04:00 UT, the Doppler shift reversal near range gate 10 .

HAIR echoes are observed for extensive periods of time during intervals of high geomagnetic activity, when the auroral oval moves to low latitudes and the precipitationenhanced E-region is located in the near ranges of the radar fields-of-view. Just such an example is presented in the two lower panels of Fig. 15, range-time-Doppler shift plots for both IW and IE radars from 00:00-24:00 UT, 1 November 2001, when both radars were operating in the normal (not myopic) scan mode, that is with $45 \mathrm{~km}$ range gates. Backscatter fills the near-range portions of the fields-of-view throughout the 24-h period. The radars observe primarily the return flow of the ionospheric convection pattern at these ranges, and the transition from the dawn convection cell to the dusk cell near local noon $(\sim 12: 00 \mathrm{UT})$ is readily apparent in the change in the predominant sense of Doppler shift in both panels. In addition, the presence of HAIR echoes in the first 5-or-so range gates is apparent throughout the interval presented. This is best observed for the IE radar between 
12:00 and 20:00 UT, but can be discerned for both radars, both pre- and post-noon.

HAIR echoes are ubiquitous, the condition for their observation being backscatter within the very nearest ranges of the radars, and an E-region critical frequency of the order of a few MHz. These two conditions often occur hand-in-hand, as the region of E-region electron density enhancement is also the region where irregularities are expected to be abundant.

\section{Summary and conclusions}

Our study allows several conclusions to be drawn, regarding the occurrence and characteristics of HF radar backscatter from the auroral electrojets:

1. E-region ionospheric irregularities arise mainly in regions of enhanced electron density, corresponding to the elevated conductivity of the electrojets.

2. The enhanced electron density results in refraction of $\mathrm{HF}$ radio waves allowing coherent backscatter to be observed from field-aligned irregularities.

3. This refraction leads to reflection of the radio waves such that ground backscatter tends to be observed at twice the range of the ionospheric backscatter.

4. At ranges close to the radar the radio waves propagate insufficiently obliquely to be refracted to orthogonality with the magnetic field, even within the enhanced electron density regions. However, backscatter is still observed from the same altitudes, though with markedly different spectral characteristics. We hypothesize that these echoes arise from high aspect angle scatter, and so term this the "high aspect angle irregularity region", or HAIR. Initial estimates suggest that aspect angles as high as $30^{\circ}$ can be observed. We suggest that such high $\alpha$ irregularities are present throughout the electrojets, but that backscatter from them is only observed where it is not masked by higher power backscatter from fieldaligned irregularities.

5. HAIR backscatter has low Doppler shifts which are consistent with poleward flow in the eastward electrojet and equatorward flow in the westward electrojet, or in other words, the expected direction of $\boldsymbol{E}$. This is consistent with linear theory that suggests that the phase motion of irregularities should rotate towards the ion drift direction as $\Psi(\alpha)$ increases. The backscatter power decreases as the estimated aspect angle increases, with an aspect sensitivity of approximately $1 \mathrm{~dB} \mathrm{deg}^{-1}$. These results contrast sharply with the reported characteristics of high-aspect angle scatter in the UHF and VHF bands, that is Doppler shifts which are proportional to, though below, the line-of-sight component of the electron drift in the $\boldsymbol{E} \times \boldsymbol{B}$ direction, sometimes exceeding the ionacoustic speed, and aspect sensitivities of some $10 \mathrm{~dB}$ $\operatorname{deg}^{-1}$. These differences can be accounted for, perhaps, by invoking a long wavelength instability mechanism, such as that proposed by Robinson and Schlegel (2000), for the generation of high-aspect angle irregularities from which HF radars can scatter.

A drawback of the current report is the simplicity of the propagation model assumed. A future study will undertake a full ray-tracing analysis to determine not only the variation of aspect angle with range, but also with azimuthal lookdirection and location of the radar with respect to the electrojet. This will give a clearer understanding of the flow angle dependence of the HAIR echoes, as well as providing a better estimate of the aspect sensitivity of the scatter. In addition, it is desirable to conduct studies employing a radar with supporting instrumentation in the near-range field-ofview which allows the E-region critical frequency to be measured, in order to confirm the relationship between $d_{\text {HAIR }}$ and foE.

Acknowledgements. CUTLASS is supported by the Particle Physics and Astronomy Research Council (PPARC grant no. PPA/R/R/1997/00256), UK, the Swedish Institute for Space Physics, Uppsala, and the Finnish Meteorological Institute, Helsinki.

The Editor in Chief thanks D. R. Moorcroft and another referee for their help in evaluating this paper.

\section{References}

Davies, K.: Ionospheric Radio, IEE Electromagnetic Waves Series 31, Peter Peregrinus Ltd., London, 1990.

Fejer, B. G. and Kelley, M. C.: Ionospheric irregularities, Rev. Geophys., 18, 401-454, 1980.

Fejer, B. G. and Providakes, J. F.: High latitude E-region irregularities: New results, Physica Scripta, T18, 167-178, 1987.

Foster, J. C., Tetenbaum, D., Del Pozo, C. F., and St.-Maurice, J.-P.: Aspect angle variations in intensity, phase velocity, and altitude for high-latitude 34-cm E-region irregularities, J. Geophys. Res., 97, 8601-8617, 1992.

Greenwald, R. A., Baker, K. B., Dudeney, J. R., Pinnock, M., Jones, T. B., Thomas, E. C., Villain, J.-P., Cerisier, J.-C., Senior, C., Hanuise, C., Hunsucker, R. D., Sofko, G., Koehler, J., Nielsen, E., Pellinen, R., Walker, A. D. M., Sato, N., and Yamagishi, H.: DARN/SuperDARN: A global view of the dynamics of highlatitude convection, Space Sci. Rev., 71, 761-796, 1995.

Haldoupis, C.: A review on radio studies of the auroral E-region ionospheric irregularities, Ann. Geophysicae, 7, 239-258, 1989.

Hall, G. E., MacDougall, J. W., Moorcroft, D. R., St.-Maurice, J.P., Manson, A. H., and Meek, C. E.: Super Dual Auroral Radar Network observations of meteor echoes, J. Geophys. Res., 102, 14 603-14 614, 1997.

Hanuise, C., Villain, J.-P., Gresillon, D., Cabrit, B., Greenwald, R. A., and Baker, K. B.: Interpretation of HF radar ionospheric Doppler spectra by collective wave scattering theory, Ann. Geophysicae, 11, 29-39, 1993.

Hunsucker, R. D.: Radio Techniques for Probing the Terrestrial Ionosphere, Springer-Verlag, Berlin, 1991.

Makarevitch, R. A., Koustov, A. V., Igarashi, K., Ohtaka, K., Ogawa, T., Nishitani, N., Sato, N., Yamagishi, H., and Yuki- 
matu, A. S.: Comparison of flow angle variations of E-region echo characteristics at VHF and HF, Adv. Polar Upper Atmos. Res., 16, 59-83, 2002.

Milan, S. E., Jones, T. B., Robinson, T. R., Thomas, E. C., and Yeoman, T. K.: Interferometric evidence for the observation of ground backscatter originating behind the CUTLASS coherent HF radars, Ann. Geophysicae, 15, 29-39, 1997.

Milan, S. E. and Lester, M.: Simultaneous observations at different altitudes of ionospheric backscatter in the eastward electrojet, Ann. Geophysicae, 16, 55-68, 1998.

Milan, S. E. and Lester, M.: Spectral and flow angle characteristics of backscatter from decametre irregularities in the auroral electrojets, Adv. Space Res., 23, 1773-1776, 1999.

Milan, S. E. and Lester, M.: A classification of spectral populations observed in HF radar backscatter from the E-region electrojets, Ann. Geophysicae, 19, 189-204, 2001.

Milan, S. E., Lester, M., Sato, N., and Takizawa, H.: On the altitude dependence of the spectral characteristics of decametrewavelength E-region backscatter and the relationship with optical auroral forms, Ann. Geophysicae, 19, 205-217, 2001.

Milan, S. E., Lester, M., and Sato, N.: Multi-frequency observations of HF radar E-region aurora, Ann. Geophysicae, 21, 761-777, 2003a.

Milan, S. E., Lester, M., Sato, N., Takizawa, H., and Villain, J.-P.: Investigation of the relationship between optical auroral forms and HF radar E-region backscatter, Ann. Geophysicae, 18, 608617,2000

Milan, S. E., Sato, N., Lester, M., Murata, Y., Shinkai, Y., Doi, H., Frey, H. U., and Saemundsson, T.: E-region echo characteristics governed by auroral arc electrodynamics, Ann. Geophysicae, in press, 2003b.

Moorcroft, D. R.: An examination of radio-auroral aspect sensitivity, Can. J. Phys., 63, 1005-1012, 1985.
Moorcroft, D. R.: A statistical study of UHF auroral backscatter at large magnetic aspect angle: A reanalysis of unpublished results from 1968, J. Geophys. Res., 101, 11 005-11 011, 1996.

Moorcroft, D. R.: Outstanding issues in the theory of radar aurora: Evidence from the frequency dependence of spectral characteristics, J. Geophys. Res., 107, 1301, doi: 10.1029/2001JA009218, 2002.

Nielsen, E.: Aspect angle dependence of backscatter intensity of 1m auroral plasma waves, J. Geophys. Res., 93, 4119-4124, 1988.

Robinson, T. R. and Schlegel, K.: The generation of non aspect sensitive plasma density irregularities by field aligned drifts in the lower ionosphere, Ann. Geophysicae, 18, 799-806, 2000.

Sahr, J. D. and Fejer, B. G.: Auroral electrojet plasma irregularity theory and experiment: A critical review of present understanding and future directions, J. Geophys. Res., 101, 26 893-26909, 1996.

Schlegel, K. and Moorcroft, D. R.: EISCAT as a tristatic auroral radar, J. Geophys. Res., 94, 1430-1438, 1989.

Villain, J. P., Greenwald, R. A., Baker, K. B., and Ruohoniemi, J. M.: HF radar observations of the $\mathrm{E}$ region plasma irregularities produced by oblique electron streaming, J. Geophys., Res., 92, 12 327-12 342, 1987.

Villain, J. P., Hanuise, C., Greenwald, R. A., Baker, K. B., and Ruohoniemi, J. M.: Obliquely propagating ion acoustic waves in the auroral E region: Further evidense of irregularity production by field-aligned electron streaming, J. Geophys. Res., 95, 78337846, 1990.

Whalen, J. A.: A quantitative description of the spatial distribution and dynamics of the energy flux in the continuous aurora, J. Geophys. Res., 88, 7155-7169, 1983. 